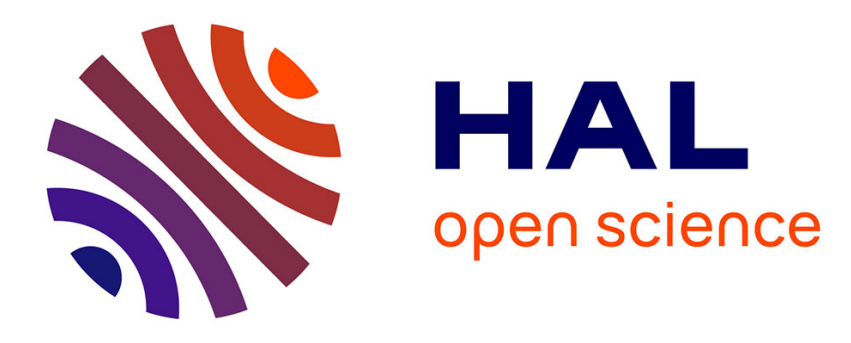

\title{
Suffusion, transport and filtration of fine particles in granular soil
}

\author{
Didier Marot, Ahmed Benamar
}

\section{To cite this version:}

Didier Marot, Ahmed Benamar. Suffusion, transport and filtration of fine particles in granular soil. Erosion of geomaterials, pp.39-79, 2012, 10.1002/9781118561737.ch2 . hal-01007547

\section{HAL Id: hal-01007547 https://hal.science/hal-01007547}

Submitted on 2 Dec 2018

HAL is a multi-disciplinary open access archive for the deposit and dissemination of scientific research documents, whether they are published or not. The documents may come from teaching and research institutions in France or abroad, or from public or private research centers.
L'archive ouverte pluridisciplinaire HAL, est destinée au dépôt et à la diffusion de documents scientifiques de niveau recherche, publiés ou non, émanant des établissements d'enseignement et de recherche français ou étrangers, des laboratoires publics ou privés. 


\title{
Suffusion, Transport and Filtration of Fine Particles in Granular Soil
}

\author{
Didier MAROT and Ahmed BENAMAR
}

\subsection{Introduction}

\subsubsection{Chapter objectives}

Flows within the interstitial pores of a soil may cause suffusion. This complex phenomenon corresponds to the process of detachment and then transport of the finest particles within the porous network. Suffusion may cause changes in porosity and can also lead to important modifications in the hydraulic and mechanical characteristics of the soil. Such modifications of the porous medium can also be the catalyst for significant instabilities at real scale of hydraulic structures.

Suffusion, i.e. the interaction between water and soil, is conditioned by the evolving and interdependent characteristics of the fluid and the solid phases. Therefore, its study is a complex matter as the phenomenon of suffusion depends on numerous geometrical, hydraulic, mineralogical, and physicochemical parameters, all in connection with solids and fluids.

The complexity of the mechanisms and phenomena at stake may cause discrepancies in the interpretation of the results of the same study [SKE 94, MON 98]. Besides, suffusion is governed by the principles of soil mechanics as well as by hydraulic principles. 
This multidisciplinary characteristic explains the duality of the main approaches, which are detailed in the relevant literature, with the aim of drawing up criteria for evaluating the initiation of this phenomenon. When suffusion takes place, a fraction of the detached particles can resettle or be filtered at the center of the porous network. This process can then locally induce a clogging. The processes of suffusion and filtration are thus indissoluble and their coupling must give a realistic account of the mechanisms that lead to the instabilities of hydraulic earth structures.

The role of a filter is to retain the particles that were suspended during a flow. The design of the filters in hydraulic works is particularly important given its role in stopping or at least slowing down erosion. The criteria used for designing these filters (especially [SHE 89]) are founded mainly on the characteristics of grain size distribution of the materials. The operating of a filter depends on the geometrical conditions (size and distribution of pores, size of particles and their concentration), hydraulic conditions (velocity, gradient), and physicochemical conditions. This process of filtration takes place through the deposition of particles on the surface of the filter or by trapping the particles in the porous medium. Experiments have enabled us to measure the indirect indicators of clogging (i.e. pressure and concentration) and also they helped us to adapt an appropriate modeling.

The first objective of this chapter is to identify the predominant suffusion parameters and present the main criteria used for evaluating the initiation of this phenomenon. By confronting several criteria, we can elaborate new criteria for the initiation and development of suffusion. Moreover, suffusion is a pathology of the filter that hydraulic works must be prevented from. The second objective is, therefore, to characterize the way filters work in order to set out the clogging conditions that can result from the properties of the filter or from the properties of the particles that need filtering. The classic criteria used for the filters are compared with the experimental results and they are discussed in this chapter.

\subsubsection{Terminology}

In relevant literature, several terms are used to describe the process of detachment and transport of fine particles within the porous network of a soil, under the action of an internal water flow.

Researcher Pavlov coined the term "suffusion" in 1898. Kovacs [KOV 81] used the term "suffusion" to describe the movement of fine particles. Undoubtedly influenced by the distinction advanced by Istomina, [KOV 81] distinguished between two types of suffusion: internal and external. The first type is characterized by the redistribution of fine particles that alters the local hydraulic conductivity of the material without causing a significant modification in the overall solid volume. 
The second type of suffusion corresponds to the pulling out and then evacuation of fine particles from the solid matrix considered; during this time, the solid volume of the matrix decreases and its hydraulic conductivity increases. However, according to Kovacs [KOV 81], the stability of the granular skeleton composed by coarser fraction of the grains remains unaffected.

To describe the transport of small particles of a type of soil, Ken and Lau [KEN 85] use the term "suffosion", and in specialized literature both "suffusion" and "suffosion" are frequently used interchangeably. The term "suffusion" is equally used in medicine to designate the blood suffusion or any other liquid that seeps through a surface. To avoid ambiguity, Chapuis and Aubertin [CHA 04] suggest that we should use the term "suffossion". Li [LI 08a] considers that "suffossion" does not describe the migrating process and shows that the term "internal stability" (or "internal instability") is generally accepted without any confusion. However, the terminology "internal stability" or "internal instability" does not mention the origin of the process and allows us to suppose a mechanical consequence of the detachment and transport of fine particles.

Following the workshop on the topic of internal erosion in April 2005 at Aussois (France), several researchers and experts of various countries have adopted a terminology that includes the term "suffusion" [FEL 07]. Consequently, this is the term that we will use in this chapter. Several types of filtration are described; however, the description of the results of the experiments regards the deep filtration that concerns hydraulic works.

\subsection{Dominant parameters that influence suffusion}

Suffusion is the result of a seepage flow in the pores of a soil. Consequently, its initiation and development depend, on the one hand, on the parameters that influence the characteristics of the fluid and solid phases and, on the other hand, on the interstitial geometry, i.e. on the exchange interface between the two phases. Garner and Fannin [GAR 10] describe the main initiation conditions for suffusion using a diagram comprising three components: material susceptibility, critical hydraulic load, and critical stress condition.

\subsubsection{Parameters that modify the geometry of the porous medium}

The detachment of fine particles and their subsequent transport throughout the porous network of the soil requires that the pore space is sufficient. This space is conditioned by the granular distribution, which, in turn, depends on the size of the grains, their form, and the fabric. Given that the flow of the water and of the 
detached particles depends on the tracking conditions of their entire run, the opening of the pores at the downstream of the considered soil volume can equally influence suffusion.

\subsubsection{Grain size distribution}

There are three main gradation curves that can be distinguished [LAF 89]: linear distribution (curves 1 and 2 in Figure 2.1), discontinuous distribution (curve 3), and, finally, upwardly concave distribution (curve 4).

In the case of the curve 2 , the coarser fraction is a minority $(<40 \%)$ and "floats" within the fine fraction. In the case of the discontinuous distribution, a series of intermediary-size grains is missing. The concave distribution consists of a poorly graded coarser fraction associated with a highly graded fine fraction. In this type of soil, the volume between the grains of the coarser fraction is higher than the volume of fine particles, thus enabling the fine particles to migrate.

The soils whose grain-size distribution curve corresponds to curves 1 or 2 are stable to suffusion [LAF 89]. The soils that are likely to suffer from suffusion are, according to [FEL 07], "internally unstable", i.e. their grain-size distribution curve is either discontinuous (curve 3) or upwardly concave (curve 4).

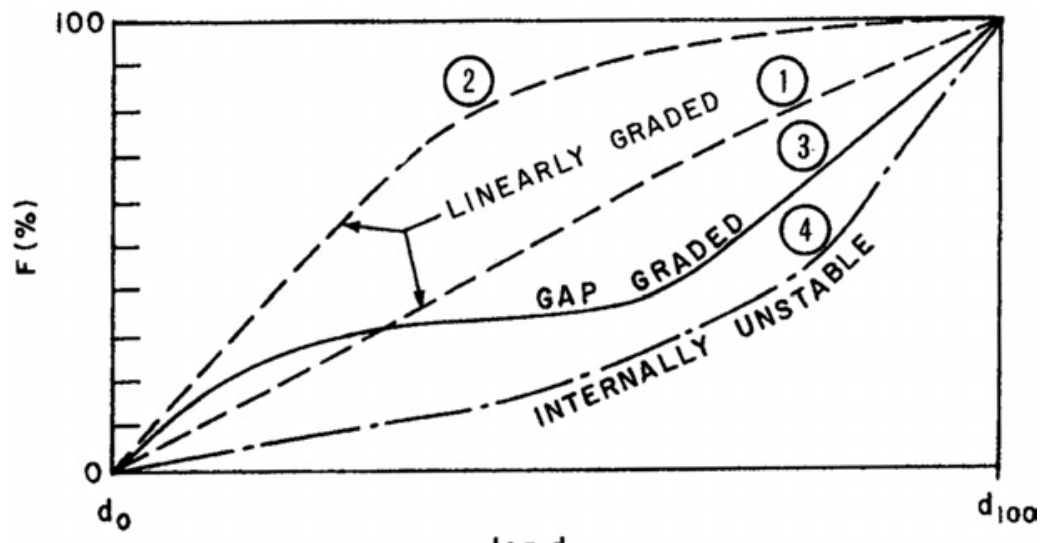

$\log d$

Figure 2.1. Classification of the grain size distribution of soils [LAF 89]

\subsubsection{Effective stress}

The Terzaghi principle consists of postulating the existence of effective stress that governs the behavior of the granular skeleton. For the same granular distribution, the modification of the effective stress can induce grain rearrangements. 
Several tests performed in oedometric conditions on unstable soils show that a rise in the effective stress causes an increase in the soils' resistance to suffusion [MOF 05]. In the same manner, when tests were carried out under isotropic confinement [BEN 08], the increase in the confinement pressure allowed for a decrease in the suffusion rate (Figure 2.2).

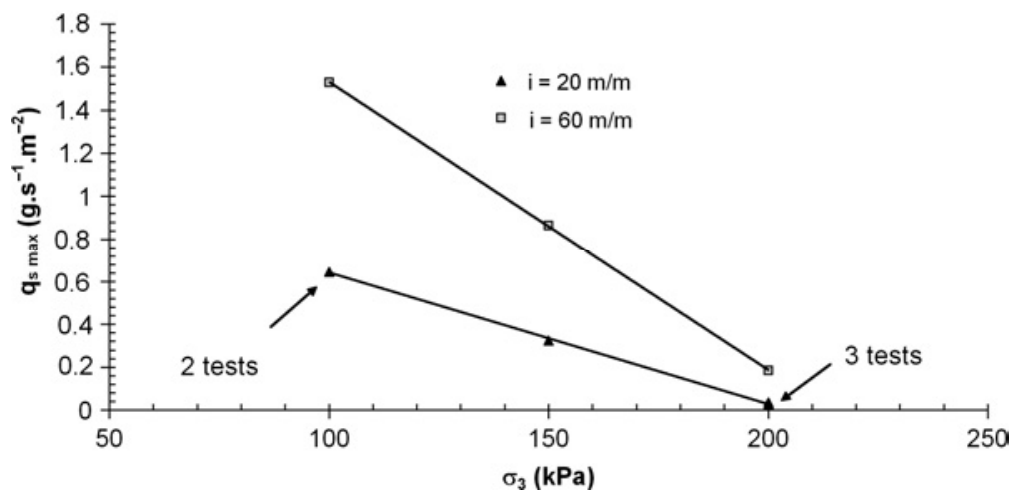

Figure 2.2. Influence of the confinement pressure on the rate of suffusion for two values of an applied hydraulic gradient (clayey sand samples) [BEN 08]

\subsubsection{Grain angularity}

Little data regarding the influence of the shape of the grains on suffusion were published. However, let us turn toward the model of the capillary tube proposed by Kovacs [KOV 81], which represents the poral space by a set of cylindrical, parallel tubes. The average diameter of the pore $d_{0}$ is calculated by using the following equation:

$$
d_{0}=4 \frac{n_{c}}{1-n_{c}} \frac{D_{h}^{c}}{\alpha_{D}}, \quad D_{h}^{c}=\left(\sum_{i} \frac{\Delta F_{i}^{C}}{D_{i}^{C}}\right)^{-1}
$$

where $n_{c}$ is the porosity of the coarser fraction, $\alpha_{D}$ is the shape coefficient, $D_{h}^{c}$ is the average diameter in the interval $i$ of the granular distribution of the coarser fraction, and $\Delta F_{i}{ }^{C}$ is the corresponding mass percentage. The porosity of the coarser fraction is expressed by $n_{c}=n+F_{n}(1-n)$, where $n$ is the porosity of the soil and $F_{n}$ is the mass percentage of the fine fraction.

One of the criteria for evaluating the initiation of suffusion is confronting the average value of the pore diameter with the size of the fine fraction (see section 2.3.1). It is worth noting that in equation [2.1] there emerges the shape coefficient 
whose values are comprised between 6 for the spherical particles and from 7 to 9 for the more angular particles [KOV 81]. Thus, grain angularity probably plays a significant role in suffusion.

\subsubsection{Downstream pore opening}

Suffusion can be described as the result of the process of surface erosion, the surface being here the surface of the pores, together with the fate of eroded particles [RED 00]. Furthermore, the distinction between internal suffusion and external suffusion [KOV 81] demonstrates the need to take into account the conditions for the evacuation of the detached particles in relation to the soil volume. This problematic is at the center of the study of filter efficiency, whether they are made up of a geotextile material or of a layer of draining soil. For cohesionless soils, [LAF 99] identifies the ideal diameter $d_{i}$ of the opening of the filter, by starting from the uniformity coefficient and from the aspect of the grain-size distribution curve. Depending on the real opening $O_{F}$ of the pores of a filter, several phenomena can be observed: suffusion, if $O_{F}>>d_{i}$, clogging if $O_{F}<<d_{i}$, and self-filtration if $O_{F} \approx d_{i}$.

Even when filters that allow the migration of all the fine particles are being used, the size of the opening of the filter's pores constitutes a parameter that can modify significantly the initiation and the development of suffusion. When testing suffusion on clayey sands, replacing a filter with a $4 \mathrm{~mm}$ opening by another filter of a $0.08 \mathrm{~mm}$ opening has enabled the critical hydraulic gradient to rise by 6 for the initiation of clay suffusion [MAR 09].

\subsubsection{Parameters that modify the physicochemical characteristics of the medium}

The physicochemical characteristics of the fluid and solid phases are crucial, particularly in the case of cohesive soils. According to Arulanandan and Perry [ARU 83], the size of the eroded aggregates is a function of either the state of flocculation or of de-flocculation. The state of flocculation or de-flocculation depends particularly on the chemical composition of the interstitial fluid, as well as on the quantity and nature of the clay.

\subsubsection{The content of sodium chlorate in the interstitial fluid}

Reddi et al. [RED 00] have carried out a series of suffusion tests on clayey sand, injecting fluids that had different concentrations of sodium chlorate. The rate of erosion by suffusion decreases when the concentration of sodium chlorate increases. There are two distinct processes that can be responsible for this decrease in the quantity of eroded particles: the decrease in the detachment of particles and the increase in the filtration of detached particles. This reduction in suffusion could 
be attributed, as is the case of surface erosion [ARU 83], to the influence of sodium chlorate on the flocculation of the soil. If the rate of sodium absorption is high, the forces of physicochemical interaction decrease, which, in turn, causes a deflocculation of the clay and a decrease in the resistance of clay particle detachment.

\subsubsection{The content and mineralogical nature of clay}

Clay particles are made up of flakes, which, in turn, are made of atoms that are arranged in tetrahedral or octahedral meshes. By studying these different packings of atoms and flakes, we can classify the different types of clay into eight categories. Among these, we should mention in particular the kaolinite group, the smectite group (mainly comprising montmorillonite), and the illite group. Their structure and their differing chemical composition give them different particle sizes and a different sensitivity to water.

For the same type of clay, several tests performed on clayey sands have shown that suffusion decreases with the increase in the clay percentage [BEN 08]. Arulanandan and Perry [ARU 83] show that the nature of the clay influences the indicator of dielectric dispersion. This indicator is obtained by measuring the electric properties of the soil and of the interstitial fluid. By taking into consideration the concentration of the interstitial fluid and the rate of sodium absorption, the dielectric dispersion indicator enables us to characterize the initiation of the detachment of clay particles [ARU 83]. Thus, as with any other type of erosion, suffusion, which depends on the conditions of detachment of particles or aggregates of particles, appears to depend on the nature of the clay, and for a given type of clay, on the clay percentage.

\subsection{Main initiation criteria for suffusion}

In what follows, we do not intend to offer a treatise on the initiation criteria, or to make up a list of all the criteria proposed in the relevant literature. For a more in-depth study of these matters, the reader can refer to [WAN 04] and [LI 08a]. The main objective here is to present the criteria that are most widely used or most suitable to characterize the initiation of suffusion. We distinguish two types of criteria: grain-size distribution and hydraulic.

\subsubsection{Grain-size distribution criteria}

The capacity for the geometric retention of a filter was initially characterized by a criterion that was generally attributed to Terzaghi and that can be expressed by the following inequality: 


$$
\frac{D_{15}}{d_{85}}<4
$$

where $D_{15}$ is the sieve size for which $15 \%$ of the weighed filter material is finer and $d_{85}$ is the sieve size for which $85 \%$ of the weighed base material is finer.

In the aim of applying this filtering criterion to the case of suffusion, Kézdi [KÉZ 79] proposed the distinction between the coarser fraction and the fine fraction in a point chosen randomly in the distribution of the grains. He considers that the coarser fraction will act as a filter for the fine fraction. The sieve size for which $15 \%$ of the weighed coarser fraction is finer (called $D_{15}^{\prime}$ ) characterizes the size of the constrictions of this fraction, and the sieve size for which $85 \%$ of the weighed fine fraction is finer $\left(d_{85}^{\prime}\right)$ is used to characterize the size of the particles in this fraction. Thus, [KÉZ 79] advances the hypothesis that the soil will be stable to suffusion if the ratio $D_{15}^{\prime} / d_{85}^{\prime}$ is well below 4 for every random division point throughout the grain size distribution. By using the same method, Sherard (quoted by [WAN 04]) proposed different numeric values for the inequality [2.2] depending on the percentage of fine particles in non-cohesive soils. Starting from the model of capillary tubes, Kovacs [KOV 81] compared the average diameter of pores $d_{0}$ (equation [2.1]) with the representative diameter of the fine fraction $d_{85}^{\prime}$. If $d_{0}<d_{85}^{\prime}$, then the soil is considered to be stable to suffusion. However, this criterion was not tested experimentally.

One of the most currently used criteria is the criterion proposed by Kenney and Lau [KEN 85]. This criterion was established based on results of tests that were carried out on samples composed of sand and gravel. It consists of determining, for each size of the particle $d$, the mass percentage $F$ of the grains, having a size that is lower than $d$, as well as the mass percentage $H$ of the grains having a size between $d$ and $4 d\left(H=F_{4 d}-F_{d}\right)$. These researchers consider that the grains smaller than $d$ can detach themselves if the soil does not have enough grains within the interval between $d$ and $4 d$. They represent the values of $H$ depending on the values of $F$ (where the obtained curve is called shape curve). Within the interval $0<F<X$, they propose that the soil be deemed unstable if its shape curve is situated completely or partially under the line represented by $H=1.3 F$ (the equation once modified in 1986 becomes $H=1.0 F$ ). In the case of soils whose coefficient of uniformity $C_{U}=d_{60} / d_{10}=3, X$ is chosen to be equal to 0.3 and if $C_{U}>3$, then $X=0.2$.

As a consequence of dry mixing tests carried out on soils composed of sand and gravel $\left(C_{U}\right.$ up to 200), Burenkova [BUR 93] assumes that a fraction of the 
small-size grains is not part of the granular skeleton of the soil if its insertion in the mixture does not entail any increase in the volume of the mixture. Conversely, if the volume of the sample rises with the addition of the fine fraction, this fraction is supposed to belong to the granular skeleton. The author suggests that the stability to suffusion depends on the ratios $h^{\prime}=d_{90} / d_{10}$ and $h^{\prime \prime}=d_{90} / d_{15}$. [BUR 93] defines the domain of the stable soils by:

$$
0.76 \log \left(h^{\prime \prime}\right)+1<h^{\prime}<1.86 \log \left(h^{\prime \prime}\right)+1
$$

Furthermore, the size of the largest particle $d_{d v}$, representing the limit between the granular skeleton and the grains sensitive to suffusion, can be estimated by:

$$
0.55 h^{\prime \prime}-1.5<\frac{d_{\mathrm{dv}}}{d_{100}}<1.87 h^{\prime \prime}-1.5
$$

Following the research works of [BUR 93] and in order to distinguish more clearly between the unstable soils and the soils stable to suffusion, Wan and Fell [WAN 08] define the probability $P$ for suffusion by:

$$
P=\frac{\exp Z}{1-\exp Z}
$$

For the mixtures of silt-sand-gravel and clay-silt-sand-gravel:

$$
Z=2.378 \log h^{\prime \prime}-3.648 h^{\prime}+3.701
$$

For the mixtures sand-gravel with less than $10 \%$ of non-plastic fine particles $(d<0.075 \mathrm{~mm})$ :

$$
Z=3.875 \log h^{\prime \prime}-3.591 h^{\prime}+2.436
$$

For the soils whose granular distribution is upwardly concave (type curve 4 in Figure 2.1) Wan and Fell [WAN 08] propose a different method as an alternative for the criteria proposed by Burenkova [BUR 93], based on the ratios $d_{90} / d_{60}$ (used to characterize the slope of the coarser fraction) and $d_{20} / d_{5}$ (characterizing the range of the fine fractions). Figure 2.3 shows $15 / \log \left(d_{20} / d_{5}\right)$ depending on $30 / \log \left(d_{90} / d_{60}\right)$, [WAN 08] discerns an area where the soils are stable to suffusion, an area where they are unstable and, finally, a transition area. 


\subsubsection{Confronting granular criteria}

Starting from the tests that dealt with sand-gravel mixtures, Li and Fannin [LI 08b] have carried out a comparative study of the criteria offered by Kézdi [KÉZ 79] and Kenney and Lau [KEN 85]. These two criteria evaluate the risk of suffusion based on the slope of the granular distribution.

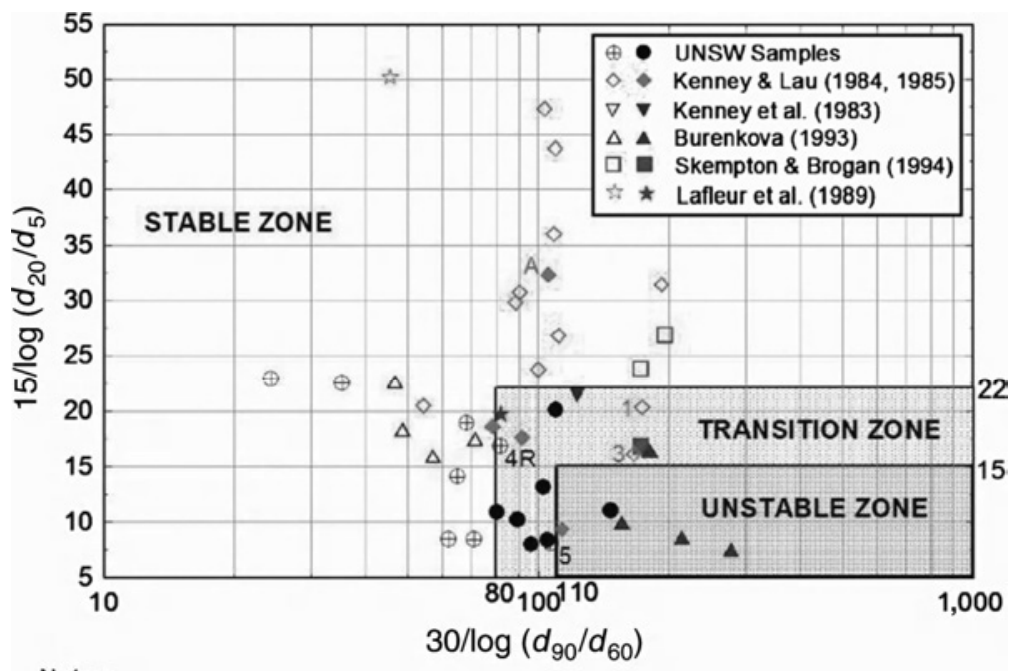

Notes:

1. Hollow symbol represents internally stable sample.

2. Solid symbol represents internally unstable sample.

Figure 2.3. Alternative method for determining the stability to suffusion [WAN 08]

According to Li and Fannin [LI 08b], the risk evaluation for suffusion made with these two criteria can be reformulated by: within an interval that corresponds to four grain diameters, the slope is lower than $15 \%$ in the case of Kézdi's criterion and lower than $\mathrm{F} \%$ in the case of the criteria proposed by Kenney and Lau [KEN 85]. The authors summarize the comparison between the two criteria by representing the increase in mass $H$ depending on the variable $F$ (Figure 2.4) for the minimal value of the ratio $H / F$.

$\mathrm{Li}$ and Fannin [LI 08b] draw the following conclusions: the two criteria converge for $F=15 \%$. In the case of soils with discontinuous grain size distribution, Kézdi's criterion seems to be more suitable for distinguishing the stable soils from the unstable soils. For the soils with continuous grain size distribution, the best results are obtained using the Kenney and Lau criterion. Kézdi's criterion is more conservative for $F<15 \%$ and, conversely, Kenney and Lau's criterion is more conservative for $F>15 \%$. 
Wan and Fell [WAN 08] have carried out 20 suffusion tests on mixtures of clay-silt-sand-gravel and silt-sand-gravel mixtures. A downward flow is generated by a hydraulic gradient of 8 . Three criteria are used for predicting the initiation of suffusion: the Sherard's criterion, the Kenney and Lau's criterion, and, finally, Burenkova's criterion.

The comparison between the experimental results and the predictions made with these three criteria shows that the Sherard's and the Kenney and Lau's criteria are quite definitely conservative. The best predictions are made using the Burenkova's criterion, and Wan and Fell [WAN 08] have proposed the calculation of probability (equations [2.3]-[2.5]) in order to improve these predictions.

We can deduce that the limits of the different grain-size distribution criteria come from the fact that several important parameters, such as the mineralogy of the material, the angularity of the grains, the effective stress force, and the viscosity of the fluid, were not taken into account. Furthermore, most of the tests were performed on cohesionless materials and did not concern cohesive soils. Finally, even if the transport of particles is geometrically feasible, the action of the hydraulic flow must be sufficient for detaching the soil particles. Thus, Kovacs [KOV 81] acknowledges that not observing a grain-size distribution criterion does not necessarily entail a strong sensitivity to suffusion, due to the contact forces between the grains that resist their detachment.

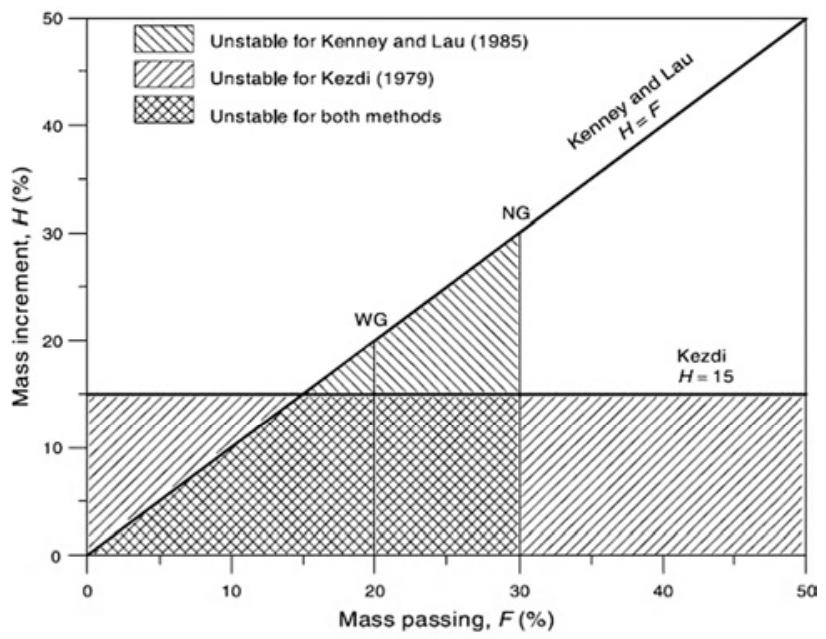

Figure 2.4. Comparison between Kézdi's criteria and Kenney and Lau's criteria [LI 08b] 


\subsubsection{Hydraulic criteria}

The action of the hydraulic flow on the grains is often described following three distinct approaches: the hydraulic gradient, the hydraulic shear stress, and the pore velocity. The critical values of these three quantities can be used to characterize the initiation of suffusion.

\subsubsection{Critical hydraulic gradient}

Under the effect of an upward water flow, the effective stress of a volume of soil can annul itself when the hydraulic gradient reaches the critical value defined by Terzaghi. This is called critical gradient of heave. This heave or blow out gradient can be expressed as the ratio between the submerged density of soil and the water density. The gradient is generally of the order of one unit.

By carrying out tests on the mixtures of sand and gravel with an upward flow, Skempton and Brogan [SKE 94] concluded that the value of the critical gradient of the initiation of suffusion, marked as $i_{\text {ch }}$, can be approximately a third or a fifth of the value of the critical heave gradient $i_{c}$. As the authors explain, the deviation between the values of the critical gradient is caused by the fact that the major part of the effective stress is supported by the granular skeleton made of gravel. They propose to express the critical suffusion gradient of the sand $i_{\text {ch }}$ by:

$$
i_{c}-i_{\mathrm{ch}}=\alpha \frac{\gamma^{\prime}}{\gamma_{w}}
$$

where $\alpha$ is a reduction factor for the effective stress, which is lower than 0.1 [SKE 94].

Monnet [MON 98] used the results of the same tests to draw up a method of evaluation for the suffusion gradient. This method aims at comparing the hydraulic conductivity $k$ of the material with the value $0.01\left(d_{15}\right)^{2}$. If $k<0.01\left(d_{15}\right)^{2}$, then the instability will be obtained by heave, and if $k>0.01\left(d_{15}\right)^{2}$, the instability will be obtained by suffusion with a critical gradient estimated by:

$$
i_{\mathrm{ch}}=0.01 \frac{\left(d_{15}\right)^{2}}{k} i_{\mathrm{c}}
$$

where $d_{15}$ is expressed in millimeters and $k$ is expressed in meters per second.

By taking into account the hydraulic conductivity, the criterion proposed by [MON 98] is equivalent to a critical Darcy velocity criterion $\left(v_{\mathrm{ch}}=k i_{\mathrm{ch}}\right)$. 


\subsubsection{Critical hydraulic shear stress}

Hillel (quoted by [RED 00]) expresses the hydraulic shear stress generated by a hydraulic flow in a tube of length $L$ and of the radius $r$ through the equation:

$$
\tau=\frac{\Delta P}{L} \frac{r}{2}
$$

where $\Delta P$ is the pressure drop throughout the tube. To generalize this approach to the study of soils, Khilar et al. [KHI 85] model the soils by using a network of capillary tubes that are parallel to the constant radius. The intrinsic permeability $K$ of the system of tubes may be expressed as follows:

$$
K=n \frac{r^{2}}{8}
$$

where $n$ is the porosity of the soil. By replacing $r$ with $K$ in the expression of the hydraulic shear stress, Red et al. [RED 00] came up with the expression:

$$
\tau=1.414 \frac{\Delta P}{L} \sqrt{\frac{K}{n}}
$$

The hypothesis that the hydraulic loading responsible for the suffusion is essentially a shear allows comparing the rate of suffusion with the hydraulic shear stress. With the aim of helping the representation of erosion rates versus the hydraulic shear stress, Shields (quoted by [ARU 83]) defines, by making a linear extrapolation, the critical hydraulic shear stress as the maximum value of the hydraulic shear stress corresponding to the absence of particle detachment.

Using equation [2.6] and by extrapolating from the curve of the maximum erosion rate versus the hydraulic shear stress, the critical value of the hydraulic shear stress was evaluated for mixtures of sand and kaolinite, consolidated under isotropic confinement of $100 \mathrm{kPa}$ [BEN 08]. Depending on the percentage of the clay, the value of the critical stress is $0.13,0.23$, and $0.32 \mathrm{~Pa}$ for $10 \%, 20 \%$, and $30 \%$ of kaolinite, respectively. Red et al. [RED 00] have compared the values of the critical hydraulic shear stress of suffusion with the values of critical hydraulic shear stress of surface erosion, measured by hole erosion test. This confrontation emphasized the fact that the critical hydraulic shear stress value of suffusion can be several orders of magnitude higher than the critical hydraulic shear stress of surface erosion. These deviations can be the result of the extremely significant difference between the exchange surfaces of the solid and the fluid, for these two types of erosion. According to [RED 00], the potential surface erosion is of the order of $5.5 \times 10^{-4} \mathrm{~m}^{2}$ 
in the case of the hole erosion tests, whereas the porosity tests using mercury reveal a potential surface erosion in the case of suffusion of $7.065 \mathrm{~m}^{2}$. It is worth noting that this very important discrepancy is, perhaps, due to the measuring method used by [RED 00] (which can be used a priori on a rigid structure). These deviations also emphasize the great difficulty in defining and measuring the critical hydraulic shear stress.

\subsubsection{Critical pore velocity}

To consider the spatial variability and the anisotropic characteristic of the hydraulic conductivity, Perzlmaier [PER 07] predicts the use of the average pore velocity for characterizing precisely the hydraulic solicitation. The average pore velocity is expressed as the ratio between the Darcy velocity and the porosity multiplied by the tortuosity.

The tortuosity is the squared ratio of the distance between two points and the length of the real course of the flow between these two points. Wittmann (quoted by [PER 07]) has evaluated the tortuosity to $2 / \pi=0.6366$. Starting from several theoretical approaches that were developed in the relevant literature, [PER 07] has calculated the critical value of the average velocity of a pore according to the size of the particles of cohesionless soils. He concludes that for particles of a size higher than $0.1 \mathrm{~mm}$, the critical pore velocity increases with the size of the particles, and for particles of a diameter lower than $0.1 \mathrm{~mm}$, the critical velocity is about $10^{-3} \mathrm{~m} / \mathrm{s}$.

For certain tests used to determine the critical velocity, it is worth noting that the initiation of the suffusion was visually detected. In the case of the detachment of silt or clayey materials, we should not exclude the possibility that this observation is not accurate enough and consequently that the value of $10^{-3} \mathrm{~m} / \mathrm{s}$ is an overestimation.

\subsubsection{Summary and final remarks}

The initiation of suffusion is governed by:

- the geometry of the porous network, which is influenced by the granular distribution, porosity, and the shape of grains;

- the hydraulic loading that was mainly modeled by the hydraulic gradient, the hydraulic shear stress, or the pore velocity.

The different confrontations carried out between the predictions of these criteria and observations emphasize some significant differences. These deviations may come from the absence of coupling between the two types of initiation criteria. 
Furthermore, the critical hydraulic shear stress values for suffusion seem very different from that of the surface erosion. These differences can be caused by the intrinsic characteristics of the different erosion processes, but the models of interpretation that were deployed can also cause these differences.

\subsection{An initiation criterion formulated using a geohydromechanical approach}

The approach proposed by Li [LI 08a] can be divided into two stages: the first stage aims at evaluating the potentiality for the initiation of suffusion by using a geometric criterion. The objective of the second stage is to evaluate the capacity of hydraulic loading to trigger the suffusion by taking into account the effective stress.

\subsubsection{Geometric criterion}

To identify the fine fraction within the granular distribution, [LI 08a] recommends choosing a point of division at the level of the discontinuity in the case of a discontinuous grain-size distribution curve, and at the level of the minimal value of the ratio $H / F$ (emerged from the criteria of Kenney and Lau) in the case of a soil with continuous grain size distribution. The fine fraction having been identified (the value of $F$ cannot be higher than $35 \%$ or else the coarser fraction floats within the fine fraction [SKE 94]), [LI 08a] calculates the average diameter $d_{0}$ of the model of the capillary tube proposed by Kovacs (equation [2.1]). Starting from the results of his tests and from several tests in relevant literature, [LI 08a] suggests that the soils whose $d_{0}<d^{\prime}{ }_{85} / 0.42$ could be considered potentially stable.

For a soil that is potentially unstable to suffusion, [LI 08a] advances a hydromechanical criterion to evaluate the hydraulic strain required for the initiation of suffusion.

\subsubsection{The hydromechanical criterion}

From the concept of stress reduction supported by the fine fraction [SKE 94], Li [LI 08a] advances the notion of the hydromechanical envelope that depends on the effective stress. This concept is expressed by:

$$
i_{c}=\frac{\alpha}{1-0.5 \alpha}\left(\bar{\sigma}_{\mathrm{vm}}^{\prime}+0.5 \frac{\gamma^{\prime}}{\gamma_{w}}\right), \quad \bar{\sigma}_{\mathrm{vm}}^{\prime}=\frac{\sigma_{\mathrm{vm}}^{\prime}}{\gamma_{w} \Delta z}, \quad \sigma_{\mathrm{vm}}^{\prime}=\frac{1}{2}\left(\sigma_{t 0}^{\prime}+\sigma_{b 0}^{\prime}\right)
$$

where $\alpha$ is the effective stress reduction factor, $\bar{\sigma}_{\mathrm{vm}}^{\prime}$ is the normalized average vertical effective stress value, $\Delta z$ is the height of the layer of soil, $\sigma_{t 0}^{\prime}$ is the 
effective stress at the top of the soil layer, and $\sigma_{b 0}^{\prime}$ is the effective stress at the base of the soil layer.

The correlation research made by [LI 08a] between $\alpha$ and three geometric indicators $\left((H / F)_{\min }, \mathrm{D}_{15}^{\prime} / d_{85}^{\prime}, d_{85}^{\prime} / d_{0}\right)$ leads to a linear correlation between $\alpha$ and the ratio $d^{\prime}{ }_{85} / d_{0}$. Thus, equation [2.7] enables us to determine the critical hydraulic gradient and, in comparison with the applied hydraulic gradient, to predict the initiation of suffusion.

\subsubsection{Summary and final remarks}

The approach proposed by Li [LI 08a] for estimating the initiation of suffusion combines a geometric criterion (founded, on the one hand, on the Kovacs model for the capillary tube diameter and, on the other hand, on the criteria proposed by Kenney and Lau [KEN 85]) and a hydromechanical envelope (mainly based on Skempton and Brogan's concept of effective stress). The consecutive stages of this framework are shown in Figure 2.5.

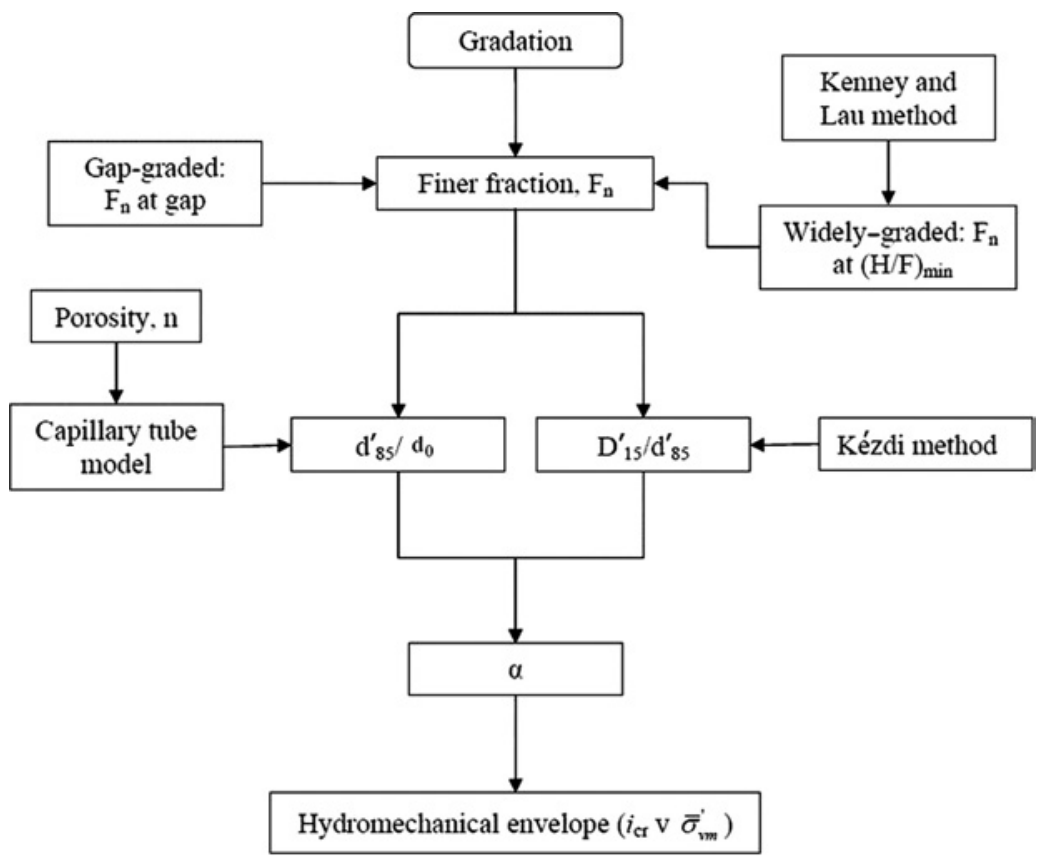

Figure 2.5. Framework for evaluating suffusion process [LI 08a] 
The critical gradient formed by Li [LI 08a] depends on the normalized average vertical effective stress, which is itself inversely proportional to the height of the layer of soil taken into consideration. The same remark can be made regarding the criterion for surface erosion proposed by Sellmeijer [SEL 88], where the critical gradient decreases depending on the length of the seepage flow [MAR 12].

This remark prompts us to further investigate the relevance of an approach based on the expression of a hydraulic gradient or on the relevance of all the laboratory tests that were carried out on a smaller scale, in relation to the large scale of the real structure. The following section discusses this scaling effect as well as the method proposed for avoiding this effect.

\subsection{The scaling effect and the energetic approach}

\subsubsection{Identifying the scaling effect}

Li [LI 08a] has carried out suffusion tests on mixtures of glass beads with discontinuous granular distribution, using two permeameters of different sizes. For the same type of tested specimen and the same applied effective stress, the values of the critical gradient to initiate suffusion can be eight times higher with the small permeameter than with the large permeameter. For a more in-depth study of this scaling effect, Mar et al. [MAR 12] have carried out a set of suffusion tests on clayey sand with different sample lengths. With the aim of controlling the condition of effective stress, the experimental device was placed on the swinging basket of a geotechnical centrifuge, thus enabling the application of different gravitational forces. Figure 2.6 represents the erosion rate that varies with the length of the tested samples and with the applied hydraulic gradient. For the same value of the hydraulic gradient and the same effective stress, the values of the suffusion rate increase linearly, depending on the length of the tested sample.

For this type of clayey sand, the values of the critical suffusion gradient obtained according to the length $H_{m}$ of the samples are $i_{c}=44 \mathrm{~m} / \mathrm{m}$ for $H_{m}=60 \mathrm{~mm}$, $i_{c}=34 \mathrm{~m} / \mathrm{m}$ for $H_{m}=90 \mathrm{~mm}$, and $i_{c}=28 \mathrm{~m} / \mathrm{m}$ for $H_{m}=120 \mathrm{~mm}$ [MAR 12]. The characterization of both initiation and development of suffusion based on the hydraulic gradient is thus perturbed by the length of the tested sample.

\subsubsection{Energetic approach}

This approach aims at determining the energy dissipated by the fluid, when it flows within the porous medium. Let $M$ be the mass of the fluid, $V$ its volume, $\rho_{w}$ 
its density, $e_{\text {int }}$ its internal energy, and $\vec{U}$ its velocity (components $u, v, w$ ). Let $S$ be the contact surface between the fluid and its environment, having the normal value $\vec{n}$. Then [REG 09] and [MAR 11] express the energy $E$ dissipated by the fluid as:

$$
\frac{d E}{d t}=\frac{\partial}{\partial t} \iiint_{\text {Volume }}\left(e_{\text {int }}+\frac{u^{2}}{2}+\vec{g} \vec{z}\right) \cdot \rho_{w} d V+\oiint_{S}\left(e_{\text {int }}+\frac{u^{2}}{2}+\vec{g} \vec{z}\right) \cdot \rho_{w}(\vec{U} \vec{n}) \cdot d S
$$

where $\vec{g}$ is the vector of the gravitational force and $z$ is the coordinates. The total energy $E$ is the sum of the mechanical energy and the energy exchanged with the environment.

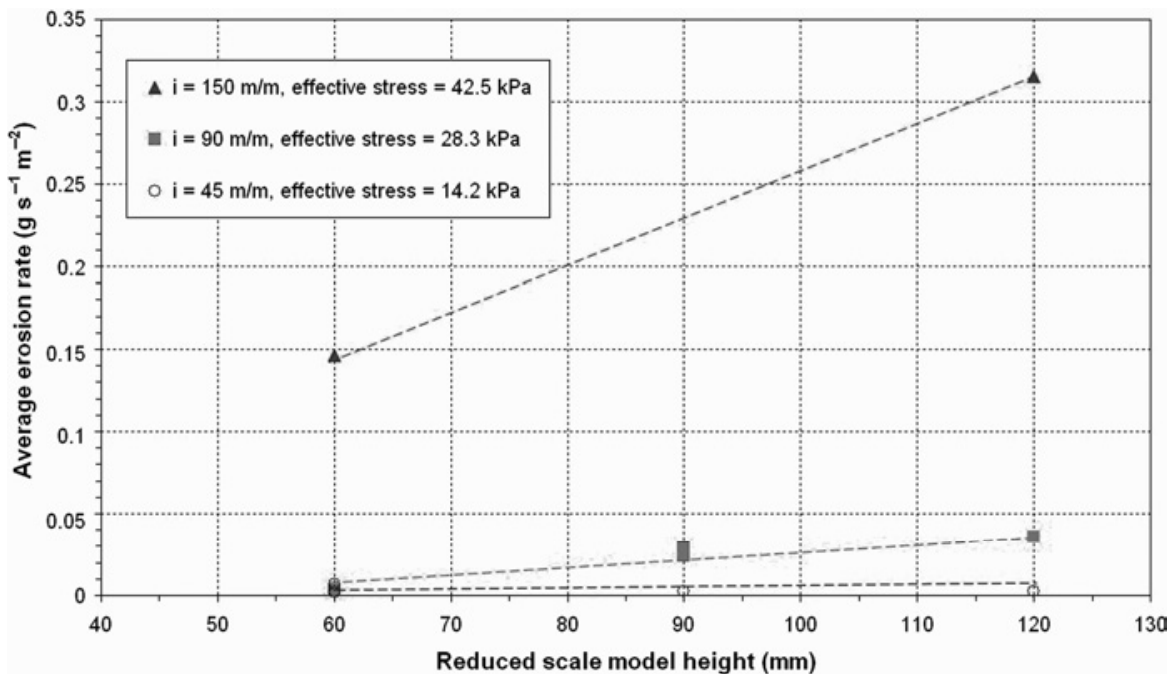

Figure 2.6. Erosion rate varying according to the height of the sample, according to the applied hydraulic gradient, as well as according to the value of effective stress [MAR 12]

In the case of suffusion, Mar et al. [MAR 12] use four hypotheses: the system is supposed to be adiabatic and isothermal, the state is supposed to be steady, and the dissipations of energy through viscosity and turbulence within the fluid are considered to be negligible in comparison with the dissipation through the contact with the soil that generates erosion. The erosion power $P_{\text {er }}$ can thus be determined by $P_{\text {er }}=Q \Delta P+\rho_{w} g L Q$, where $Q$ is the flow rate and $\Delta P$ is the pressure drop between the upstream and downstream of a sample of length $L$. 
The average erosion rate increases linearly, depending on the average erosion power, and independently of the length of the sample (Figure 2.7(a)). For every test, the total mass of the eroded clay can be plotted according to the energy dissipated by erosion, which, in turn, is calculated by time integrating the erosion power (Figure 2.7(b)). The total mass of the eroded clay is linearly correlated to the dissipated energy and this correlation is unperturbed by the scaling effect.

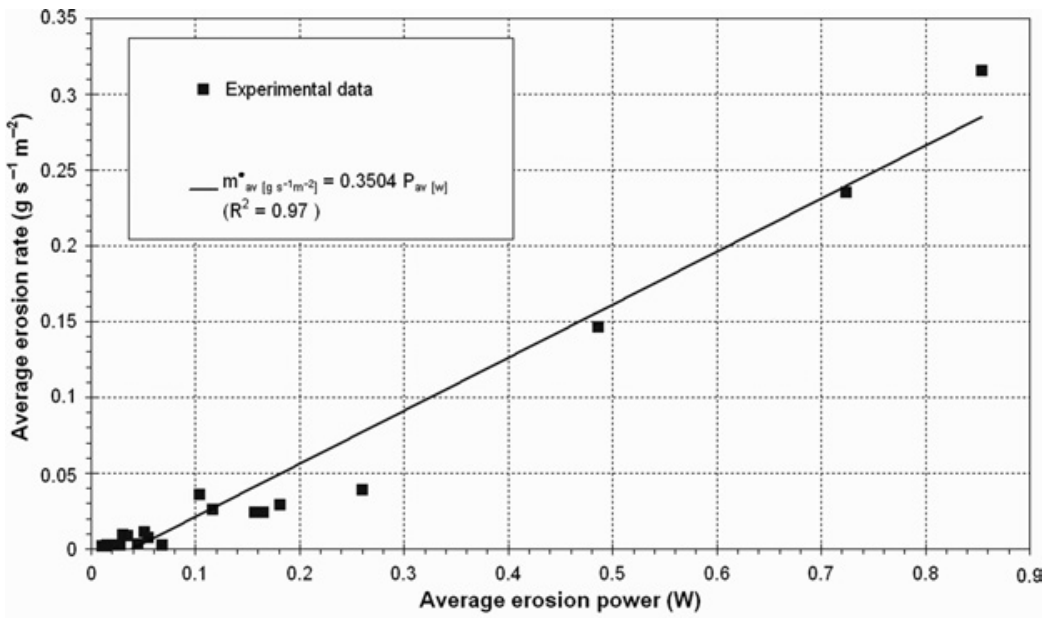

a)

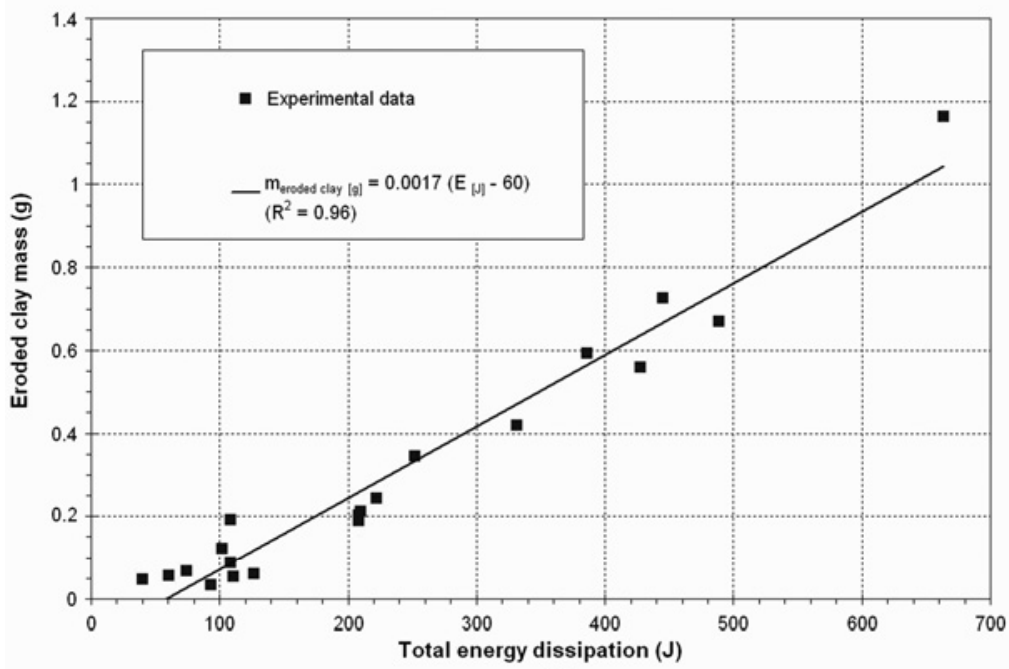

b)

Figure 2.7. (a) Erosion rate, varying with the average erosion power and (b) total mass of eroded clay, varying with the dissipated energy [MAR 12] 


\subsubsection{Summary}

Several experimental campaigns of suffusion [LI 08a, MAR 12] and of surface erosion experiments [SEL 88] emphasize the fact that the value of the critical hydraulic gradient to initiate erosion decreases with the size of the tested sample. The energetic approach that consists of determining the power of erosion and the energy dissipated by the fluid allows us to avoid this scaling effect.

The different approaches that were previously detailed have a bearing on the characterization of the initiation of suffusion as well as on the estimation of the average erosion rate and on the estimation of the total mass eroded by suffusion. Recent research has centered on the simulation of the coupling between suffusion and filtration; this relationship was emphasized through experiments that we will present in more detail below.

\subsection{Coupling the phenomena of suffusion and filtration-clogging}

Several tests that were carried out on mixtures of sand and clay [RED 00, BEN 08, MAR 09] show that hydraulic conductivity decreases throughout the phenomenon of suffusion (Figure 2.8). This decrease in hydraulic conductivity is attributed to a clogging of some pores caused by the clay particles that were detached and then redeposited, or that were detached and then filtered in the soil.

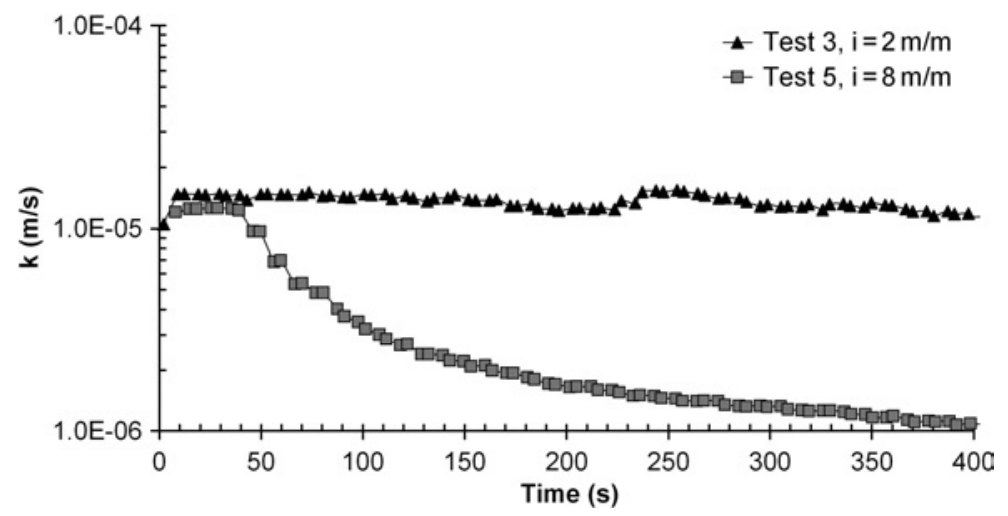

Figure 2.8. Evolution throughout time of the hydraulic conductivity without erosion (test 3) and in the presence of clay suffusion (test 5) [MAR 09]

Furthermore, in the suffusion tests involving a constant global hydraulic gradient, which were carried out on cohesionless materials with discontinuous grain size distribution [SAI 11], an increase in the local hydraulic head was measured 
before the massive detachment of fine particles. This local increase in the pressure is attributed to a filtration of certain particles that were detached in the upstream of the area in question. However, in the case of non-cohesive materials, the filtration is localized in very restricted areas on the tested sample. The global hydraulic conductivity is not significantly modified.

Suffusion, i.e. the process of detachment and then transport of fine particles, is completely dependent on the fate of eroded particles. A fraction of the fine particles will be able to migrate toward a much more porous medium, while other particles will be filtered in the soil. The amplitude of the incidence of this type of filtration on hydraulic conductivity varies with the nature of the fine particles and the porosity of the granular skeleton. In the case of cohesive fine particles, a decrease in hydraulic conductivity can be measured, and in the case of non-cohesive fine particles, the effect of the filtration can be limited to an increase in the interstitial pressures, increase that is much localized.

The next section deals with a more in-depth analysis of filtration.

\subsection{Processes causing filtration}

The clogging of pores in a permeable granular filter by eroded fine particles can drastically reduce its drainage capacity and lead to the instability of the hydraulic earth structures. The granular filters are subjected to a long accumulation of fine particles, and must remain permeable in order to guarantee the safety of the hydraulic structure. The efficiency of the process of filtration depends on various parameters, which can be classified into two categories: geometrical parameters (porosity, shape and size of grains and particles, concentration of suspension, and density) and hydraulic parameters (flow velocity, Reynolds number, and Péclet number). We can distinguish between four types of filtration: the bed filtration (retention of particles whose dimensions are higher than a fixed size), tangential filtration (the flow of the suspension running parallel to the surface of the filtering medium), the filtration with cake formation (i.e. very dense suspensions formed through autofiltration), and deep filtration (diluted suspensions; the particles of a size lower than that of the pores that infiltrate deep into the porous medium and that deposit at different depths). Predicting the importance of the transport of particles is difficult because of the complexity of the processes that govern the detachment, the transport, and the deposition of the particles in a porous medium. We can discern four types of retention sites: the surface sites, the crack sites, the constriction sites (in the upstream of the shrinkage of pores whose size is lower than that of the particles), and the cavern sites (Figure 2.9). 


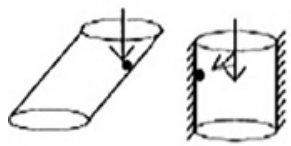

Surface sites

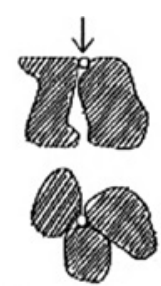

Crevices sites

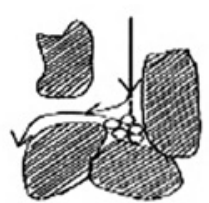

Straining sites

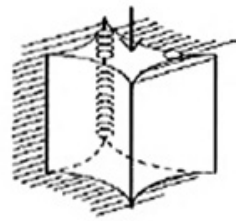

Cavern sites

Figure 2.9. Different types of retention sites [HER 70]

\subsubsection{Background knowledge}

The absence of a filter in an earthen hydraulic structure can cause its failure by erosion, and the use of a poorly adapted filter can cause either clogging or a massive migration of particles [FOS 00]. If the initiation of internal erosion is the first condition of its existence, the absence or the unsuitability of the filter to stop the transport of particles is the second condition. The criterion based on the ratio between the opening of the sieve that allows for $15 \%$ of the material of the filter to pass through it $\left(D_{15 F}\right)$ and the opening of the sieve that allows $85 \%$ of the soil particles to be filtered $\left(d_{85 B}\right)$ is often used by engineers when they design the filters [SHE 89]. However, the porosity and the permeability are also significant parameters and the geometrical criteria are complemented by a hydraulic criterion. Although the deep filtration is a well-known technology, the mechanisms responsible for the retention of particles are not yet fully comprehended.

The deep filtration is a complex process in view of the different mechanisms at stake [HER 70]. We can discern two major types of forces: hydromechanical forces and physicochemical forces. The experiments carried out in two columns on porous media with latex spheres have shown that the diameter of particles plays a major part in the transport of particles [CUM 99]. Significant research was carried out regarding the movement of particles in suspension as well as the mechanical blockage (or straining), interception, sedimentation, or diffusion [MCD 86]. For coarser particles, those that are larger than $10 \mu \mathrm{m}$, the hydrodynamic, gravitational, and inertial effects are predominant, whereas for the small particles whose dimension varies between 0.1 and $1 \mu \mathrm{m}$ [GOH 99], all the forces and mechanisms are accounted for.

The results obtained by Khilar and Fogler [KHI 84] on the colloidal materials explain the interaction between the chemical conditions and the hydrodynamic conditions in the processes of retention and release of fine particles. The remobilization arises below a critical concentration of salt and above a critical flow 
velocity. The studies carried out on the detachment and filtration of clayey particles in a porous medium have shown that the trapping of particles does not take place below a certain threshold value of the concentration of particles.

The trapping of fine particles in the constrictions is partly due to the direct interception and partly due to the size exclusion [MAY 05]. The effect of the physicochemistry on the filtration of colloidal particles in a saturated porous medium was studied by [TUF 04], where the trapping efficiency of the collector is modeled as the sum of the contributions of different transport mechanisms.

The research works of [FRE 99] on the physicochemical effects have shown that they determine not only the distribution of the deposition in the porous medium but also the clogging process. The importance of the relationship of pore solution in the medium was largely studied for the colloidal particles that present the adequate surface properties [GRO 01, TOS 09], and was less studied for the soil particles [KHI 84, BLU 05].

The first criteria for deep filtration [HER 70] are founded on the ratio between the dimensions of the suspended particles $\left(d_{p}\right)$ and the grains of the porous medium $\left(d_{g}\right)$, and they predict the blockage of particles if the diameter of these particles is 0.05 times higher than the average diameter of the medium grain $d_{50}\left(d_{p} / d_{50}>0.05\right)$. Bradford et al. [BRA 04] have shown that even for a ratio of $d_{p} / d_{50}$ equal to 0.002 , the trapping of particles can well take place. Other authors [SIL 95] stress the importance of not only the effect of the size of particles but also the importance of their shape and nature. Angular particles cause their deposition and a fall in the permeability that is more important than in the case of spherical particles. According to [GHI 94], the (spherical) particles are systematically blocked starting from a threshold of $d_{p} / d_{g}=0.155$. On the contrary, when this ratio is below 0.065 , the retention of particles is negligible. The studies carried out on the pore scale advance a capturing model with two kinetics: the transport of particles up to the interface with the grain of the porous medium, and then the attachment of the particles on the surface of the collector. Probabilistic models [RED 05] founded on a capillary flow were developed to quantify the capturing of particles in a porous medium. Several factors, such as the velocity and the pressure, can lead to a detachment of particles that were previously deposited in the medium.

\subsubsection{Theoretical background}

The basis for the understanding of the process of flow and particle transport in the porous media was largely developed from experimental and theoretical studies on the macroscopic models, where the coupling at the pore scale is not often 
measured or observed directly. The experimentation was focused on the measuring of the variables and average properties of the medium, such as the porosity, the permeability, the fluid pressure, and the concentration of particles in the laboratory reactors. There are two types of forces that act on the retention of particles in a porous medium: hydromechanical forces and physicochemical forces.

The hydrodynamic forces are the following:

- The gravitational pull: it causes the sedimentation of the particle and it is expressed as:

$$
F_{G}=\frac{1}{6} \pi d_{p}^{3}\left(\rho_{p}-\rho\right) g
$$

where $\rho_{p}$ is the volume mass of the particle, $\rho$ is the volume mass of the water, and $g$ is the gravitational constant.

- The drag force: in the case of a Newtonian fluid and a laminary flow that acts upon a spherical particle, this force is expressed by:

$$
F_{D}=32 \mu a_{p} U_{\text {ap }}
$$

where $U_{\text {ap }}$ is the velocity of the fluid on a radial distance that is equal to the radius of the particle $a_{p}$, and $\mu$ is the viscosity of the water.

- The inertial force: this centrifugal force is approached by:

$$
F_{G}=\frac{1}{3} \pi d_{p}^{3}\left(\rho_{p}-\rho\right) \frac{u^{2}}{d_{g}}
$$

where $u$ is the average interstitial velocity.

The physicochemical forces are the following:

- The Van der Walls forces: these are intermolecular forces of attraction of an electromagnetic origin, which act on very short distances $(10 \mathrm{~nm})$; for a sphere that interacts with a plane, these forces are expressed by:

$$
F_{\mathrm{vdw}}=\frac{\pi H d_{p}}{12 h^{2}}
$$

where $H$ is the Hamaker constant that characterizes every surface $\left(10^{-19}\right.$ to $10^{-20} \mathrm{~J}$ for every condensed phase) and $h$ is the distance between the particle and the solid grain. 
- The electrostatic forces of the double electric layer: in the specialized literature, there have been several formulations of the electrostatic force, starting from the resolution of the Poisson-Boltzman equation, with well-defined boundary conditions [KHI 84]; in the case of the interaction between a plain surface and a sphere, the appropriate expression is the following:

$$
F_{d l}=2 \pi \varepsilon a_{p} \kappa \exp (-\kappa h)\left[2 \psi_{p} \psi_{g}-\left(\psi_{p}^{2}+\psi_{g}^{2}\right) \frac{\exp (-\kappa h)}{(1-\exp (-2 \kappa h))}\right]
$$

where $\varepsilon$ is the dielectric constant of the fluid, $\kappa$ is the Debye-Huckel parameter that characterizes the extension of the load of counter-ions in the solution, $\psi_{p}$ and $\psi_{g}$, respectively, are the surface potential of the particle and of the wall of the porous medium.

These different forces will compete against one another in the porous medium and they contribute to the retention or remobilization of the particles. Several retention mechanisms depend on the nature of these suspended particles (mineralogy, shape, size, etc.) of the properties of the fluid ( $\mathrm{pH}$, ionic force, etc.) and of the properties of the medium (the nature of its components, size and distribution of the pores, shape of the grains, etc.). These mechanisms, which are illustrated in Figure 2.10, are the following:

- The mechanical blockage (or straining): this mechanism depends directly on the relation between the diameter of the grains $d_{g}$ and the diameter of the suspended particles $d_{p}$; the blockage is the most dominant mechanism for the ratio $d_{g} / d_{p} \leq 10$ [MCD 86] and leads to clogging; it can be ignored if $d_{g} / d_{p}>20$. More recent experiments involving well-calibrated porous media have reached a ratio of $d_{50} / d_{p}>200$ from which the mechanical blockage can be neglected [BRU 05]. However, this ratio evolves according to the number of particles that arrive at the entrance of a constriction. The results of these recent experiments carried out on micromodels show that the only particles that are not blocked are the particles with a diameter lower than 1.5 times the diameter of pores [KEL 04];

- Interception: in view of its size, a particle may collide with the collector and can reside inside the convergent walls of the pore surface; this trapping is linked to the ratio of the dimensions of the grain and the particle called interception parameter.

- Sedimentation: the sedimentation velocity of these particles can be calculated using Stokes' law; sedimentation can contribute to the deposit of particles in the flows of low velocity. 
- Diffusion: in the absence of electrostatic forces, diffusion causes the retention of particles on the collectors; this mechanism for transport and deposition can be quantified using the Brownian diffusion coefficient $D=K_{B} T /\left(3 \pi \mu d_{p}\right)$, where $K_{B}$ is the Boltzman constant and $T$ is the temperature .

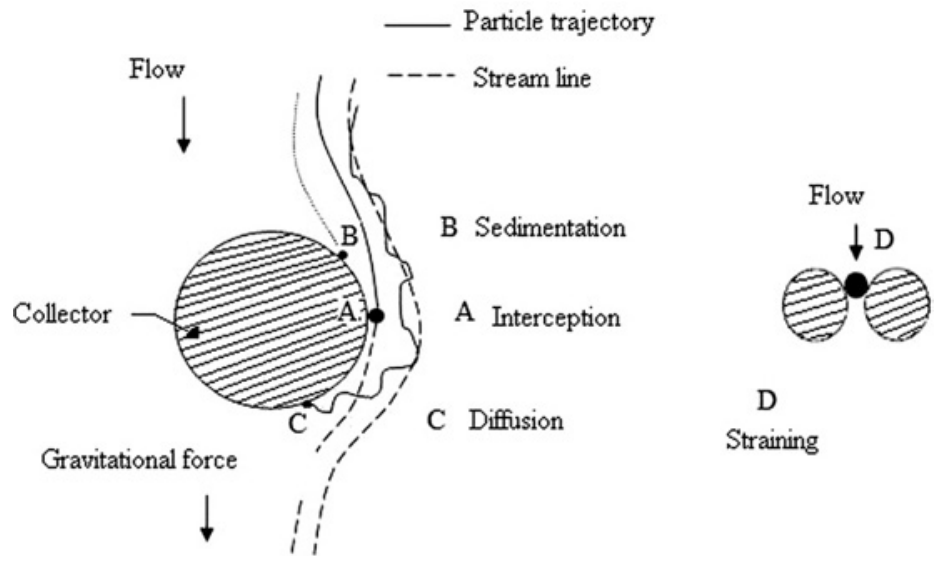

Figure 2.10. Mechanisms of transport and trapping of a particle using a spherical collector [MCD 86]

\subsection{Filtration modeling}

\subsubsection{Modeling in a continuous medium}

\subsubsection{Homogeneous medium}

There are two approaches that can describe the transport of particles in a porous medium. The first is the macroscopic approach, which is based on a mass balance at the level of each volume element of the porous medium and consists of following the average concentration in each point. The deposition of particles is described by a filtration coefficient. The second is the microscopic approach, which is founded on the individual study of different processes of interaction that could develop in the medium. In this approach, the capture and the detachment efficiency (or the adsorption/desorption) is described not only in terms of empirical transfer coefficient but also in terms of the contributions brought by the different transport mechanisms (section 2.7.2). In steady-state flow, by ignoring the dispersion and the diffusion mechanisms (by considering a clean porous medium), the empirical model describing the proportion of the deposited particles as a function of the first order of concentration is expressed by the so-called filtration model: 


$$
\frac{\partial C}{\partial x}=-\lambda C
$$

where $C$ is the concentration of particles in the fluid phase and $\lambda$ is the filtration coefficient $\left(L^{-1}\right)$. The solution of equation [2.8] describes an exponential decrease in concentration. Starting from the restitution curve obtained through filtration tests (step-input injection of the suspension of a concentration $C_{0}$ in a column of length $L$ and the measuring of the outlet concentration $\left.C_{L}\right)$, we obtain $\lambda=-\ln \left(C_{L} / C_{0}\right) / L$.

The efficiency model for capturing a simple collector emerges from the microscopic considerations and it expresses the relation between the deposition kinetics of the particle and its velocity $u$ of approaching a collector:

$$
\eta=\frac{I_{d}}{u C \pi a_{c}^{2}}
$$

where $I_{d}$ is the global mass retention flux, obtained by adding the local fluxes of particles on the entire surface of the collector, and $a_{c}$ is the collector radius.

The particular deposition can be described by two processes: the transport of particles in suspension toward the surface of the grain defined by the frequency of collisions with the collector and the attachment of the particles or the efficiency of the collision. In the presence of the repulsive forces of the double electric layer, the capture efficiency is multiplied by an efficiency collision factor $\alpha$ :

$$
\eta=\alpha \eta_{0}
$$

According to [TUF 04], the kinematics of the deposition of particles calculated by the sum of the individual contributions of the different transport mechanisms (diffusion, interception, and sedimentation) agrees very well with the one calculated using the solution of the equation of convection diffusion. The capture efficiency $\eta_{0}$ can be expressed by:

$$
\eta_{0}=\eta_{D}+\eta_{G}+\eta_{I}
$$

where $\eta_{D}$ is the capture efficiency by Brownian diffusion, $\eta_{G}$ is the contribution of the gravity, and $\eta_{I}$ describes the retention by interception.

The collision efficiency $\alpha$ represents the fraction of the particles attached to the grains following the collisions. Given that the classical theories are unable to predict 
the efficiency of the collision or of attachment, the experiments carried out in the reactors are often used to determine $\alpha$ by:

$$
\alpha=-\frac{2}{3} \frac{d_{g}}{(1-n) L \eta_{0}} \ln \left(\frac{C}{C_{0}}\right)
$$

where $L$ is the length of the porous medium, $n$ is its porosity, and $C$ is the concentration of the particles at the outlet of the medium. The relation between the deposition kinetics coefficient $K_{\text {dep }}$ and the capture efficiency of a collector $\eta_{0}$ is [RYA 96]:

$$
K_{\text {dep }}=\frac{3}{2} \frac{(1-n)}{d_{g} n} U \alpha \eta_{0}
$$

where $U$ is the Darcy velocity. $K_{\text {dep }}\left[\mathrm{T}^{-1}\right]$ is thus a coefficient that links the variation of the particle concentration to the concentration of the medium.

In the case of deep filtration, the filtration coefficient $\lambda$ is linked to $\eta_{0}$ through the relation:

$$
\lambda=\frac{3}{2} \frac{(1-n)}{d_{g}} \alpha \eta_{0}
$$

\subsubsection{Heterogeneous medium}

To give a more realistic description of the heterogeneity effect on the flow and the transport in the porous media, a model of a medium with double porosity (macropores and micropores) is described by [BAI 95]:

$$
\left\{\begin{array}{l}
D \frac{\partial^{2} C_{1}}{\partial x^{2}}-u \frac{\partial C_{1}}{\partial x}=f \frac{\partial C_{1}}{\partial t}+K\left(C_{1}-C_{2}\right) \\
-\frac{u}{b} \frac{\partial C_{2}}{\partial x}=(1-f) \frac{\partial C_{2}}{\partial t}-K\left(C_{1}-C_{2}\right)
\end{array}\right.
$$

where $C_{1}$ and $C_{2}$ are the concentrations of particles in the macropores and micropores, $D$ is the dispersion coefficient in the macropores, $u$ is the average velocity of the interstitial fluid in the region of the macropores, $f$ is the fraction of porosity contained in the macropores, $K$ is the celerity of the mass transfer between the stagnant fluid and the flowing fluid, $x$ is the distance starting from the source, 
and $b$ is the inverse of the factor of convection intensity, used to account for the difference in the flow velocity between the two regions of porosity.

The convection-dispersion model with the two regions mobile-immobile model (MIM) is founded on a hypothesis of fractioning the water in mobile area $\theta_{m}$ and immobile area $\theta_{\mathrm{im}}$. The particles are then transported by convection-dispersion within the mobile water, and they diffuse at the interface mobile water/immobile water according to a kinetics of the first order. The non-reactive transport is thus described by the equations:

$$
\left\{\begin{array}{l}
\theta_{m} \frac{\partial C_{m}}{\partial t}+\theta_{\mathrm{im}} \frac{\partial C_{\mathrm{im}}}{\partial t}=\theta_{m} D_{m} \frac{\partial^{2} C_{m}}{\partial x^{2}}-q \frac{\partial C_{m}}{\partial x} \\
\theta_{\mathrm{im}} \frac{\partial C_{\mathrm{im}}}{\partial t}=\alpha\left(C_{m}-C_{\mathrm{im}}\right)
\end{array}\right.
$$

where $\theta_{m}$ and $\theta_{\mathrm{im}}$, respectively, are the mobile and immobile water contents, and $C_{m}$ and $C_{\mathrm{im}}\left[\mathrm{ML}^{-3}\right]$, respectively, are the concentrations in the fractions of mobile and immobile water; $D_{m}$ is the dispersion coefficient in the mobile fraction $\left[\mathrm{L}^{2} \mathrm{~T}^{-1}\right]$, $q$ is the exchange kinetics coefficient, and $\alpha$ is the exchange coefficient between the two fractions $\left[\mathrm{T}^{-1}\right]$. This model has three parameters: $\theta_{m}, D_{m}$, and $q$.

\subsubsection{Convection model-dispersion with deposition kinetics}

We can distinguish between three main migration mechanisms of the elements of the solution in a porous medium: convection (advection), molecular diffusion, and kinematic dispersion. Convection is described by the principle of mass conservation:

$$
\frac{\partial C}{\partial t}=-u \nabla C
$$

where $u$ is the average pore velocity, also called effective velocity.

The diffusion coefficient $D_{0}\left[\mathrm{~L}^{2} \mathrm{~T}^{-1}\right]$ of a concentration $C$ of particles is given by Fick's second law:

$$
\frac{\partial C}{\partial t}=D_{0} \frac{\partial^{2} C}{\partial x^{2}}
$$

Kinematic dispersion is a mixture of phenomena essentially linked to the heterogeneities of the microstructure of the medium that creates fluctuations in the flow velocities, regardless of the observation scale. This phenomenon results in a 
dilution in the concentration plume. The kinematic dispersion $D$ and the molecular diffusion $D_{0}$ are generally described by the same equation [2.9], and the global coefficient is called hydrodynamic dispersion coefficient $D$.

Taking into account the stock variation of the matter (deposit or release), the molecular transport throughout granular porous media and under conditions of a saturated and uniform flow can be described, in the one-dimensional case, by:

$$
\frac{\partial C}{\partial t}=D_{L} \frac{\partial^{2} C}{\partial x^{2}}-u \frac{\partial C}{\partial x}-\frac{\rho}{n_{0}} \frac{\partial S}{\partial t}
$$

In the hypothesis of first-order kinetics, the source term is expressed as follows:

$$
\frac{\rho}{n_{0}} \frac{\partial S}{\partial t}=K_{\mathrm{dep}} C-\frac{\rho}{n_{0}} K_{r} S
$$

where $D_{L}$ is the longitudinal hydrodynamic dispersion coefficient of particles $\left[\mathrm{L}^{2} \cdot \mathrm{T}^{-1}\right], u$ is the average velocity of the pores of the particles $\left[\mathrm{L} \cdot \mathrm{T}^{-1}\right], \rho$ is the apparent density of the solid matrix $\left[\mathrm{M} \cdot \mathrm{L}^{-3}\right], n_{0}$ is the porosity $\left[\mathrm{L}^{3} \cdot \mathrm{L}^{-3}\right]$, and $K_{\text {dep }}$ and $K_{r}$ are the deposition and release kinetics coefficients of the particles $\left[\mathrm{T}^{-1}\right]$, respectively. These equations describe the evolution of the concentration of the particles in suspension and the mass of the deposited particles per mass unit of the porous medium $S\left[\mathrm{M} \cdot \mathrm{M}^{-1}\right]$, which varies according to the transport distance and to the period of time. The initial deposition and release kinetics of the particles are assumed to follow the first-order kinetics law.

If we neglect the release (small quantity of particles available), equation [2.10] becomes:

$$
\frac{\partial C}{\partial t}=D_{L} \frac{\partial^{2} C}{\partial x^{2}}-u \frac{\partial C}{\partial x}-K_{\mathrm{dep}} C
$$

Equation [2.11] is no longer valid if the previously deposited particles influence the deposit kinetics of the newly injected particles [KRE 99]. We then obtain a deposition kinetics that depends on the concentration of particles in the aqueous phase and thus on the fraction of the porous medium available for the deposition $\Psi$. The latter is linked to the retention capacity through the porous medium, $X_{\max }$, by:

$$
\psi=\frac{X_{\text {max }}-S}{X_{\max }}
$$


The differential equation with the second-order deposit kinetics is then expressed as follows:

$$
\frac{\rho}{\omega_{0}} \frac{\partial S}{\partial t}=K_{\mathrm{dep}} \psi C-\frac{\rho}{\omega_{0}} K_{r} S
$$

The modeling through a macroscopic approach [COM 01] has led us to define the convection-dispersion equations by considering two first-order deposition kinetics sites (reversible-irreversible):

$$
\frac{\partial S_{s}}{\partial t}=\frac{\partial S_{\mathrm{rev}}}{\partial t}+\frac{\partial S_{\mathrm{irr}}}{\partial t}, \quad \frac{\partial S_{\mathrm{rev}}}{\partial t}=K_{\mathrm{dep}} C-K_{r} C_{\mathrm{rev}}, \quad \frac{\partial S_{\mathrm{irr}}}{\partial t}=K_{\mathrm{dep}} C
$$

where $S_{\text {rev }}$ is the mass of reversibly deposited particles per unit pore volume and $S_{\text {irr }}$ is the mass of irreversibly deposited particles per unit pore volume.

\subsection{Confrontation between the laboratory filtration tests and the modeling}

To investigate the dispersion and deposition parameters of particles, the most frequent laboratory tests are carried out in columns that recreate the granular medium where the identified suspensions are injected. The tracing tests are usually carried out with a step-input suspension injection, but other experiments with a pulse injection are also carried out with different particles [COM 01, MAS 02, BEN 05]. This type of test, where we inject a small suspension volume, allows us to avoid a likely deposition of particles that would modify the porosity of the medium. In the case of a pulse injection ( $C_{0} d t=m / Q$ ), where $m$ is the mass of particles, adequate boundary conditions provide an analytical solution of equation [2.11]:

$$
C(t, x)=\frac{m x}{Q \sqrt{4 \pi D_{L} t^{3}}} \exp \left(-K_{\mathrm{dep}} t\right) \exp \left[-\frac{(x-u t)^{2}}{4 D_{L} t}\right]
$$

\subsubsection{Validation of the model in the case of suspended particles}

The convection-dispersion model was used to simulate the transport and deposition of silt particles (having a modal dimension of $14 \mu \mathrm{m}$ ) in a column (Figure 2.11) filled with coarse sand or with glass beads [BEN 05, AHF 09]. The sand has an angular shape with $d_{50}=2.35 \mathrm{~mm}$ and the glass beads have a uniform size of $3 \mathrm{~mm}$. We 
will first use the device with pulse injections of suspended materials, and then we determine, by using the convection-dispersion model (transfer function of equation [2.47]), the residence time and the dispersion coefficient of the medium as well as the deposition kinetics. Having introduced the tracer in the porous medium, these tracing experiments (dissolved tracer or particular tracer) consist of measuring the time variation of the concentration (breakthrough curve or restitution curve). Figure 2.12(a) shows the measured signal (dimensionless parameters $C_{r}$ and $t_{r}$ ) with a pulse injection of silt particles and the value calculated with equation [2.12]. The shape of this type of curve characterizes the transport and the deposition in a granular medium. A homogeneous medium is characterized by a symmetrical curve, and the spread of the curve indicates a strong dispersion of the medium. A tail at the end of the curve and the maximum record (curve "peak") arising earlier than the average residence time indicates the presence of stagnant areas that are in a slow exchange with the part of the mobile fluid (heterogeneous medium). The curve shows a slight tail in view of the heterogeneity of the sand (angular grains) that may contain sites of low porosity.

\subsubsection{Results and preferential flow}

\subsubsection{Influence of the geometrical and hydrodynamic parameters}

The restitution of fine particles at the outlet of the reactor is better for the glass beads when this medium has a permeability that is lower than that of the gravel. Thus, this result shows that the overall parameters of a porous medium do not suffice for characterizing the process of mass transfer and that the organization of the poral space is a defining element. The dispersion in the porous medium is a parameter that characterizes the slowing down of the transport of particles in relation to the carrying fluid. The values obtained for the two media are very similar and they increase with velocity. The deposition kinetics of the sand is more important in view of the more abundant constrictions than in the medium made of glass beads. It increases with the flow velocity up to a "critical" value of the velocity, and then it starts decreasing. From this behavior, we can induce that there are two different flow regimes where the geometric effects and the hydrodynamic effects do not have the same importance.

\subsubsection{Size exclusion}

The comparison of the filtration between the dissolved tracer (fluorescein) and the suspended particles shows that the latter is retained shorter in the porous medium. The restitution of particles takes place before the restitution of the tracer (Figure 2.12(b)); the particles are thus transported faster than the dissolved tracer 
(velocity ratio close to 1.1). This transport behavior was associated with the phenomenon of size exclusion or the phenomenon of hydrodynamic chromatography. In fact, the coarser particles "sampled" the highest velocities in the column because they cannot approach the walls due to their size. This behavior shows the importance of the shape of the grains in the filter and its efficiency, and that the average values of the grain-size distribution parameters as well as the hydraulic parameters do not suffice for characterizing the transport and the filtration.

\subsection{Filtration and clogging}

\subsubsection{Phenomenological laws}

The phenomenological models take into account the equations for mass conservation, a kinetic equation that describes the rate of particle retention and an equation that characterizes the evolution of the pressures. These models are not founded on a physical modeling of the particle retention mechanisms. In the case where the diffusion is negligible (pure convection), the equation of mass conservation is written as follows:

$$
\frac{\partial \sigma}{\partial t}+u \frac{\partial C}{\partial x}=0
$$

where $C$ is the mass concentration of suspended particles, $u$ is the pore velocity, and $\sigma$ is the retention of particles (volume of captured particles per unit filter volume).

Using the kinetic law described in equation [2.8], we can express retention as follows:

$$
\frac{\partial \sigma}{\partial t}=\lambda u C
$$

The variation in permeability can be determined by using the Kozeny-Carman model. The modeling of pressure drop is expressed from the nonlinear variation of the hydraulic gradient according to the retention of particles [LEC 93]:

$$
\frac{i}{i_{0}}=\frac{1}{(1-j \sigma)^{m}}
$$

where $j$ and $m$ are constants in the model, which vary according to the type of filter. 


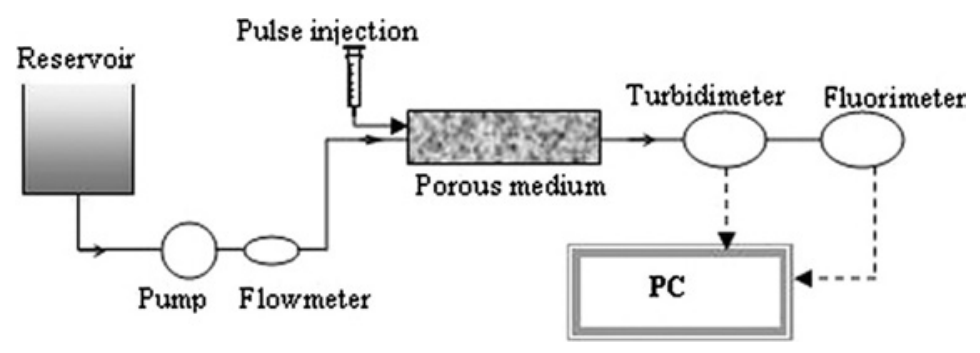

Figure 2.11. Layout of the tracing device in a laboratory reactor
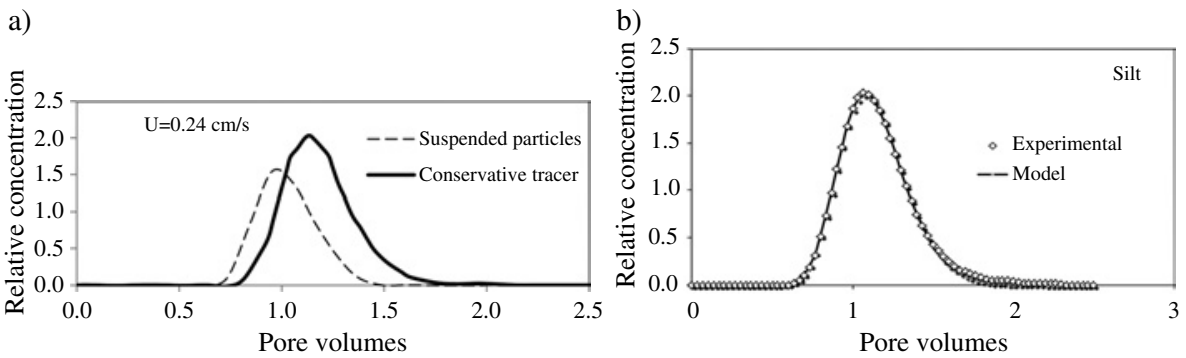

Figure 2.12. (a) Modeling of the breakthrough curve using the convection-dispersion model and (b) facilitated transport of particles in relation to the solute

In the case of a step-input injection of suspended particles in a porous medium, under certain boundary conditions, the convection-dispersion equation with deposition kinetics accepts an analytical solution given by:

$$
C(t, x)=\frac{C_{o}}{2}\left[\exp \left(\frac{(u-v) x}{2 D_{L}}\right) \operatorname{erfc}\left(\frac{(x-u t)}{2 \sqrt{D_{L} t}}\right)+\exp \left(\frac{(u+v) x}{2 D_{L}}\right) \operatorname{erfc}\left(\frac{(x+v t)}{2 \sqrt{D_{L} t}}\right)\right]
$$

where $v=u\left(1+4 K_{\mathrm{dep}} D_{L} / u^{2}\right)$.

\subsubsection{Physicochemical aspects}

The macroscopic and microscopic modelings of filtration have shown that it is difficult to separate the capture mechanisms from the physicochemical processes. The modeling of the transport and filtration of particles must integrate the coupling of the concentration of suspended particles into the ionic force of the solution. The study of the influence of the $\mathrm{pH}$ of the fluid on the transport of particles 
(Figure 2.13(a)) shows that the retention of particles decreases when the $\mathrm{pH}$ increases. The acid medium thus favors the retention of particles (deposition kinetics). This property is emphasized by the decrease in the thickness of the double electric layer (Derjaguin, Landau, Verwey, Overbeek - DLVO theory) when the medium becomes acid. However, this effect of the $\mathrm{pH}$ is more important for the clay particles than for the silt particles. The deposition kinetics coefficient increases with the ionic force (Figure 2.13(b)) up to a certain value, and then it stagnates or decreases slightly. This behavior shows the existence of a critical salt concentration (CSC) starting from which the attraction effect of the suspended particles by the grains of the porous medium is inversed. The silt particles show a more important deposition kinetics because of their size (the effect of the gravitational pull). The influence of the salinity on the two types of particles is somewhat similar.

\subsubsection{Filtration and clogging}

The analysis of the breakthrough curve, in the case of step-input injection of particles, allows us to deduce the retention parameter of the granular medium (filter). Figure 2.14(a) shows a typical breakthrough curve, expressed according to the number of pore volumes of injected suspension. This typical curve shows a first phase that corresponds to a transient filtration regime, before reaching the permanent regimen where the proportion of filtered particles remains constant (constant kinetics). The increasing part characterizes the dispersion and the plateau is characteristic of the deposition kinetics, obtained by $K_{d}=-\left(1 / t_{c}\right) \ln \left(C / C_{0}\right)$, where $t_{c}$ is the convection time measured on the breakthrough curve (Figure 2.14(b)) and corresponding to a value of $C / C_{0}$ equal to 0.5 . The results obtained for the clogging of filters quantify the variation of indirect parameters such as the pressure gradient and the permeability. The tests carried out with a constant flow rate or a constant hydraulic load show an exponential decrease in permeability with time (Figure 2.14(a)). The reduction in permeability is more drastic in the sand (stronger retention of particles) than in the glass beads, starting from a certain quantity of particles inserted into the filter. The reduction in permeability is important for a certain length of the filter. In the case of filtration with a constant hydraulic load, this reduction in permeability is the most important (clogging) and it concerns a less important length of the filter. The volume of the injected suspension influences the clogging kinetics and the distribution of the retention of particles. The decrease in permeability of the filter is not linear and so it is approached by a power law.

The application of the usual criteria has shown that the filter (sand 160-315 $\mu \mathrm{m}$ ), for which $D_{15 \mathrm{~F}} / d_{85 \mathrm{~B}}=11$, seems insufficiently permeable for avoiding the overpressures (the gradient reaches the value of 38 and the reduced permeability reaches the value of $90 \%$ ), even if it filters all the eroded particles. Since the filter 
must retain the eroded particles while remaining permeable to water, this second property is not maintained for this type of filter. A priori, for all the filters whose $D_{15 \mathrm{~F}} / d_{85 \mathrm{~B}}<11$, the permeability criterion is no longer satisfied and the Sherard's criterion [SHE 89] is no longer verified. The results show that this classical criterion $\left(D_{15 \mathrm{~F}} / d_{85 \mathrm{~B}}<9\right)$ of the filters' design is not appropriate while maintaining the permeability for the used soils. The Terzaghi filtration rule is not verified but filtration occurs. The geometric criterion that is defined according to the solid flow cannot be dissociated from the potential mass of the erodible particles (the concentration introduced in the filter). The evolution of the permeability of the filter according to the ratio $D_{15 \mathrm{~F}} / d_{85 \mathrm{~B}}$ shows threshold values for this ratio (between 15 and 20) below which permeability falls and clogging quickly occurs.

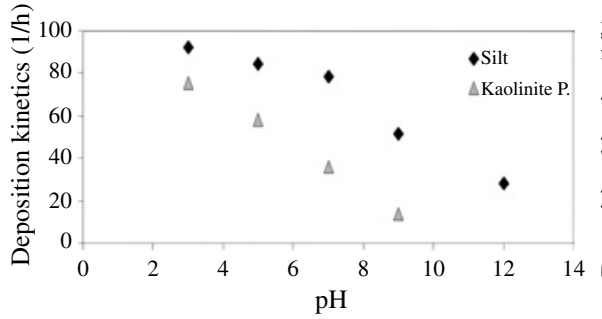

a)

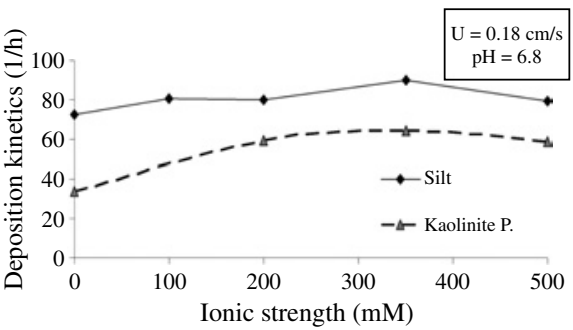

b)

Figure 2.13. Influence of (a) the $\mathrm{pH}$ and (b) the ionic force on the deposition kinetics of particles in a granular medium

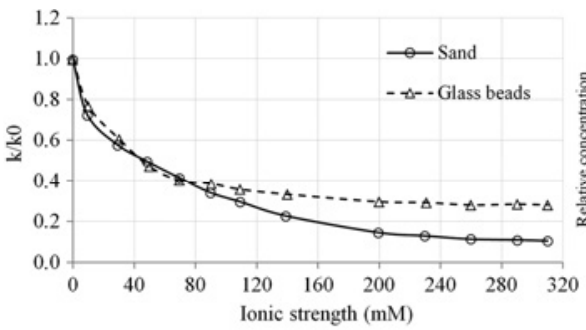

a)

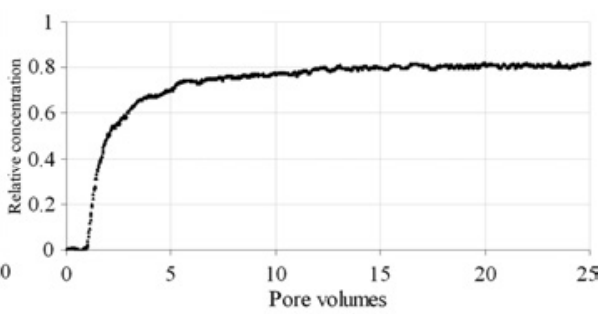

b)

Figure 2.14. (a) Breakthrough curve of the continuous injection of particles and (b) reduction of the measured permeability in a filtration test

\subsection{Conclusion}

Suffusion and filtration are two coupled processes that are governed by the geometry of the porous network, the physicochemical interactions between the solid and the fluid phases, as well as by the hydrodynamic conditions. Several evaluation 
criteria for suffusion have been developed, starting from the study of the grain size distribution of the soil. The confrontation of the estimations obtained using these grain-size distribution criteria has emphasized the limits of these criteria and the necessity to associate the analysis of the grain size distribution with the evaluation of hydraulic loading. The hydraulic approach consists of evaluating the generated load by the fluid flow in order to initiate the suffusion. This evaluation is mainly carried out by expressing the critical value of the hydraulic gradient, the shearing stress, or the pore velocity. However, different experimental campaigns have pointed out the presence of a scaling effect that perturbs the hydraulic approach based on the expression of a global hydraulic gradient. The energetic approach that consists of determining the erosion power and the energy dissipated by the fluid is not affected by this scaling effect and allows us to estimate the average erosion rate as well as the total mass of eroded particles. Throughout the development of suffusion, we can measure a localized increase in interstitial pressures, as well as a decrease in permeability. These modifications of the hydraulic characteristics are attributed to a filtration at the center of the soil of a fraction of the detached particles. The main results of the study of filtration with a pulse injection concern the evolution of the deposition function of the particles with the geometric and hydrodynamic parameters. The introduction through a step-input injection of a significant quantity of fine particles throughout a filter, under controlled conditions, allows us to quantify the indirect indicators of clogging. The use of different grainsize distribution filters has allowed us to emphasize the influence of various parameters on the kinetics of filtration. The comparison of the results with the classical criteria for the design of filters has shown the insufficiency of these criteria and the importance of the hydraulic criterion. The filters must be sufficiently fine and also the pores between the grains must be small in order to retain the eroded particles that flow through the filters. However, these filters must also be made up of grains that are coarse enough to allow the setup of flow through the filter and prevent the formation of hydraulic overpressures. The results obtained show that the filtration and the clogging depend on the concentration of the suspension in particles and of the flow conditions. The results indicate an exponential distribution of particle retention with the depth of the filter and a clogging localized mainly in a thin layer at the inlet of the filter.

\subsection{Bibliography}

[AHF 09] Ahfir N.-D., Benamar A., Wang H.Q., Alem A., "Influence of internal structure and medium length on transport and deposition of suspended particles: a laboratory study", Transport in Porous Media, vol. 76, pp. 659-668, 2009.

[ARU 83] Arulanandan K., Perry E.B., "Erosion in relation to filter design criteria in earth dams", Journal of Geotechnical Engineering, vol. 109, no. 5, pp. 682-696, 1983. 
[BAI 95] Bai M., Bouhroum F., Civan F., Roegiers J.C., "Improved model for solute transport in heterogeneous media", Journal of Petroleum Science and Engineering, vol. 14, pp. 65-78, 1995.

[BEn 05] Benamar A., Wang H.Q., Ahfir N.-D., Alem A., Massei N., Dupont J.-P., "Effets de la vitesse d'écoulement sur le transport et la cinétique de dépôt de particules en suspension en milieu poreux saturé”, C. R. Geoscience, vol. 337, pp. 497-504, 2005.

[Ben 08] Bendahmane F., Marot D., Alexis A., "Parametric study of suffusion and backward erosion”, Journal of Geotechnical and Geoenvironmental Engineering, vol. 134, no. 1, pp. 57-67, 2008.

[BLU 05] Blume T., Weisbrod N., Selker J.S., "On the critical salt concentrations for particle detachment in homogeneous sand and heterogeneous Hanford sediments", Geoderma, vol. 124, pp. 121-132, 2005.

[BRA 04] Bradford S.A., BetTAhar M., SimuneK J., VAn Genuchten M.T., "Straining and attachment of colloids in physically heterogeneous porous media", Vadose Zone Journal, vol. 3, pp. 384-394, 2004.

[BrU 05] Brusseau M.L., Oleen J.K., Santamaria J., Cheng L., Orosz-Coghlan P., Chetochine A.S., Blanford W.J., Rykwalder P., Gerba C.P., "Transport of microsporidium Encephalitozoon intestinales Spores in sandy porous media", Water Research, vol. 39, pp. 3636-3642, 2005.

[BUR 93] BurEnKova V.V., "Assessement of suffusion in noncohesive and graded soils", Proceedings of the 1st Conference on Geo-Filters, Karlsruhe, Germany, Balkema, Rotterdam, The Netherlands, pp. 357-360, 1993.

[CHA 04] Chapuis R.P., Aubertin M., "Reply to the discussion by D. Hansen on the use of the Kozeny-Carman equation to predict the hydraulic conductivity of soils", Canadian Geotechnical Journal, vol. 41, pp. 994-996, 2004.

[COM 01] COMPÈre F. Porel G., Delay F., "Transport and retention of clay particles in saturated porous media. Influence of ionic strength and pore velocity", Journal of Contaminant Hydrology, vol. 49, nos. 1-2, pp. 1-21, 2001.

[CUM 99] CUMBIE D.H., MCKAY L.D., "Influence of diameter on particle transport in a fractured shale saprolite", Journal of Contaminant Hydrology, vol. 37, pp. 139-157, 1999.

[FEL 07] FELL R., FRY J.J. (eds), Internal Erosion of Dams and Their Foundations, Taylor \& Francis, 2007.

[FOS 00] Foster M., Fell R., Spannagle M., "The statistics of embankment dam failures and accidents", Canadian Geotechnical Journal, vol. 37, pp. 1000-1024, 2000.

[Fre 99] Frey J.M., Schmitz P., Dufreche I., Gohr Pinheiro I., "Particle deposition in porous media: analysis of hydrodynamic and weak inertial effects", Transport in Porous Media, vol. 37, pp. 25-54, 1999. 
[GAR 10] GARNER S.J., FANNIN R.J., "Understanding internal erosion: a decade of research following a sinkhole event", The International Journal on Hydropower \& Dams, vol. 17, pp. 93-98, 2010.

[GHI 94] GhidaGlia C., Filtration en profondeur de particules, Doctoral Thesis, University of Paris VI, 1994.

[GOH 99] Gohr Pinheiro I., Schmitz P., Houi D., "Particle capture in porous media when physico-chemical effects dominate", Chemical Engineering Science, vol. 54, pp. 3801-3813, 1999.

[GRO 01] Grolimund D., Elimelich M., Borcovec M., "Aggregation and deposition kinetics of mobile colloidal particles in natural porous media", Colloids and Surfaces A: Physicochemical and Engineering Aspects, vol. 191, pp. 179-188, 2001.

[HER 70] Herzig J.P., LeClerC D.M., LE GoFF P., "Flow of suspension through porous media, application to deep bed filtration", Industrial and Engineering Chemistry, vol. 62, pp. 8-35, 1970.

[KEL 04] Keller A.A., Sirivithayapakorn S., Chrysikopoulos C., "Early breakthrough of colloids and bacteriophage MS2 in a water saturated sand column", Water Resources Research, vol. 40, no. W08304, p. 11, 2004.

[KEN 85] KENNEY T.C., LAU D., "Internal stability of granular filters", Canadian Geotechnical Journal, vol. 22, pp. 215-225, 1985.

[KÉZ 79] KÉzDI, Soil Physics: Selected Topics, Elsevier Scientific Publishing Co, Amsterdam, 1979.

[KHI 84] KHILAR K.C., FogleR H.S., "The existence of a critical salt concentration for particle release”, Journal of Colloid Interface Science, vol. 101, pp. 214-224, 1984.

[KHI 85] KhILAR K.C., Fogler H.S., Gray D.H., "Model for piping-plugging in earthen structures”, Journal of Geotechnical Engineering, vol. 111, no. 7, pp. 833-846, 1985.

[KOV 81] Kovacs G., Seepage Hydraulic, Elsevier Scientific Publishing Co, Amsterdam, 1981.

[KRE 99] Kretzschmar R., Borkovec M., Grolimund D., Elimelech M., "Mobile subsurface colloids and their role in contaminant transport" (review paper), Advances in Agronomy, vol. 66, pp. 121-194, 1999.

[LAF 89] Lafleur J., MLynareK J., Rollin A.L., "Filtration of broadly graded cohesionless soils”, Journal of Geotechnical Engineering, vol. 115, no. 12, pp. 1747-1768, 1989.

[LAF 99] LAFLEUR J., "Selection of geotextiles to filter broadly graded cohesionless soils", Geotextiles and Geomembranes, vol. 17, pp. 299-312, 1999.

[LEC 93] LECLERC D., DoDDS J., "De la filtration des colloïdes à la modélisation des milieux poreux", Entropie, vol. 29, no. 180, pp. 21-28, 1993. 
[LI 08a] Li M., Seepage induced failure of widely graded cohesion-less soils, PhD Thesis, Department of Civil Engineering, University of British Columbia, Vancouver, Canada, 2008.

[LI 08b] Li M., FANNIN J., "Comparison of two criteria for internal stability of granular soil", Canadian Geotechnical Journal, vol. 45, pp. 1303-1309, 2008.

[MAR 09] Marot D., Bendahmane F., Rosquoet F., Alexis A., "Internal flow effects on isotropic confined sand-clay mixtures", Soil \& Sediment Contamination, an International Journal, vol. 18, no. 3, pp. 294-306, 2009.

[MAR 11] Marot D., REgazzoni P.L., WAHL T., "An energy based method for providing soil surface erodibility rankings", Journal of Geotechnical and Geoenvironmental Engineering, vol. 137, no. 12, pp. 1290-1294, 2011.

[MAR 12] Marot D., Le V.D., Garnier J., Thorel L., Audrain P., "Study of scale effect in an internal erosion mechanism", European Journal of Environmental and Civil Engineering, vol. 16, no. 1, pp. 1-19, 2012.

[MAS 02] Massei N., Lacroix M., Wang H.Q., DupONT J.-P., "Transport of particulate material and dissolved tracer in a highly permeable porous medium: comparison of the transfer parameters", Journal of Contaminant Hydrology, vol. 57, pp. 21-39, 2002.

[MAY 05] MAYS D.C., HUNT J.R., "Hydrodynamic aspects of particle clogging in porous media", Environmetal Science and Technology, vol. 39, pp. 577-584, 2005.

[MCD 86] MCDOWELl-Boyer L.M., HunT J.R., SiTAR N., "Particle transport through porous media”, Water Resources Research, vol. 22, no. 13, pp. 1901-1921, 1986.

[MOF 05] MOFFAT R., Experiments on internal stability of widely graded cohesionless soils, $\mathrm{PhD}$ Thesis, Department of Civil Engineering, University of British Columbia, Vancouver, Canada, 2005.

[MON 98] MONNET A., "Boulance, érosion interne, renard. Les instabilités sous écoulement", Revue Française de Géotechnique, vol. 82, pp. 3-10, 1998.

[PER 07] Perzlmaier S., "Hydraulic criteria for internal erosion in cohesionless soil", in Fell R., FRY J.J. (eds), Internal Erosion of Dams and Their Foundations, Taylor \& Francis, pp. 179-190, 2007.

[RED 00] Reddi L.N., LeE I., Bonala M.V.S., "Comparison of internal and surface erosion using flow pump test on a sand-kaolinite mixture", Geotechnical Testing Journal, vol. 23, no. 1, pp. 116-122, 2000.

[RED 05] RedDi L.N., XiaO M., HaJRA M.G., LeE I.M., "Physical clogging of soil filters under constant flow rate versus constant head", Canadian Geotechnical Journal, vol. 42, pp. 804-811, 2005.

[REG 09] Regazzoni P.L., Confrontation et analyse d'érodimètres et caractérisation de la sensibilité à l'érosion d'interface, Doctoral Thesis, University of Nantes, 2009.

[RYA 96] RYAn J.N., Elimelech M., "Colloid mobilization and transport in groundwater", Colloids and Surfaces A, vol. 107, pp. 1-56, 1996. 
[SAI 11] SaIl Y., Marot D., Sibille L., Alexis A., "Suffusion tests on cohesionless granular matter", European Journal of Environmental and Civil Engineering, vol. 15, pp. 799-817, 2011.

[SEL 88] SellmeIJeR J.B., On the mechanism of piping under impervious structures, $\mathrm{PhD}$ Thesis, Delft University of Technology, The Netherlands, 1988.

[SHE 89] SHERARD J.L., DUNNIGAN L.P., "Critical filters for impervious soils", Journal of Geotechnical Engineering, July 1989.

[SIL 95] Silliman S.E., "Particle transport through two-dimensional, saturated porous media: influence of physical structure of the medium", Journal of Hydrology, vol. 167, pp. 79-98, 1995.

[SKE 94] SKempton A.W., BRogan J.M., "Experiments on piping in sandy gravels", Géotechnique, vol. 44, no. 3, pp. 440-460, 1994.

[TOS 09] Tosco T., Tiraferri A., Sethi R., "Ionic strength dependent transport of microparticles in saturated porous media: modeling mobilization and immobilization phenomena under transient chemical conditions", Environmental Science and Technology, vol. 43, pp. 4425-4431, 2009.

[TUF 04] TufENKJI N., Elimelech M., "Correlation equation for predicting single-collector efficiency in physicochemical filtration in saturated porous media", Environmental Science and Technology, vol. 38, pp. 529-536, 2004.

[WAN 04] WAN C.F., FELL R., Experimental investigation of internal instability of soils in embankment dams and their foundations, UNICIV report no. 429, University of New South Wales, School of Civil and Environmental Engineering, 2004.

[WAN 08] WAN C.F., FELL R., "Assessing the potential of internal instability and suffusion in embakment dams and their foundations", Journal of Geotechnical and Geoenvironmental Engineering, vol. 134, no. 3, pp. 401-407, 2008. 